\title{
ON SOME FAST WELL-BALANCED FIRST ORDER SOLVERS FOR NONCONSERVATIVE SYSTEMS
}

\author{
MANUEL J. CASTRO, ALBERTO PARDO, CARLOS PARÉS, AND E. F. TORO
}

\begin{abstract}
The goal of this article is to design robust and simple first order explicit solvers for one-dimensional nonconservative hyperbolic systems. These solvers are intended to be used as the basis for higher order methods for one or multidimensional problems. The starting point for the development of these solvers is the general definition of a Roe linearization introduced by Toumi in 1992 based on the use of a family of paths. Using this concept, Roe methods can be extended to nonconservative systems. These methods have good wellbalanced and robustness properties, but they have also some drawbacks: in particular, their implementation requires the explicit knowledge of the eigenstructure of the intermediate matrices. Our goal here is to design numerical methods based on a Roe linearization which overcome this drawback. The idea is to split the Roe matrices into two parts which are used to calculate the contributions at the cells to the right and to the left, respectively. This strategy is used to generate two different one-parameter families of schemes which contain, as particular cases, some generalizations to nonconservative systems of the well-known Lax-Friedrichs, Lax-Wendroff, FORCE, and GFORCE schemes. Some numerical experiments are presented to compare the behaviors of the schemes introduced here with Roe methods.
\end{abstract}

\section{INTRODUCTION}

The goal of this article is to design robust and simple first order explicit numerical schemes for solving Cauchy problems for a general class of one-dimensional nonconservative hyperbolic systems. Hyperbolic systems of conservation laws with source terms and/or nonconservative products are particular cases. A number of models of this type have been introduced in fluid dynamics to serve as simplified models of multiphase or multilayer flows. The theory introduced in [10] is used here to define the weak solutions of the system. This theory allows one to give a sense to the nonconservative terms of the system as Borel measures provided that a Lipschitz-continuous family of paths is prescribed in the space of states.

We consider here the discretization of the systems by means of numerical schemes which are path-conservative in the sense introduced in 20. The concept of a path-conservative method, which is also based on a prescribed family of paths,

Received by the editor November 24, 2008 and, in revised form, May 11, 2009.

2000 Mathematics Subject Classification. Primary 74S10, 65M06, 35L60, 35L65, 35L67.

Key words and phrases. Nonconservative hyperbolic systems, finite volume method, approximate Riemann solvers, coefficient-splitting schemes, GFORCE method, well-balanced schemes, high order methods.

This research has been partially supported by the Spanish Government Research project MTM2006-08075. The numerical computations have been performed at the Laboratory of Numerical Methods of the University of Málaga. 
provides a generalization of the conservative schemes introduced by Lax for systems of conservation laws which are also based on the choice of a family of paths. The simple first order path-conservative methods to be designed here are intended to be used as the basis for higher order methods for one and two-dimensional problems.

In [6] it has been proved that, in general, the numerical solutions provided by a path-conservative numerical scheme converge to functions which solve a perturbed system in which an error source-term appears on the right-hand side. The appearance of this source-term, which is a measure supported on the discontinuities, has been first observed in [15] when a scalar conservation law is discretized by means of a nonconservative numerical method. Nevertheless, in certain special situations the convergence error vanishes for finite difference methods: this is the case for systems of balance laws. Moreover for more general problems, even when the convergence error is present, it may only be noticeable for very fine meshes, for discontinuities of large amplitude, and/or for large-time simulations; see [6] for details.

The family of generalized Roe schemes introduced in [28] constitutes a particular case of path-conservative numerical methods. Although the schemes of this family are robust and have good well-balanced properties (see, for instance, [2, 8], 21, 20]) they also present, as their conservative counterpart, some drawbacks:

- their implementation requires the explicit knowledge of the eigenstructure of the intermediate matrices (see [23]); when their analytic expression is not available, the eigenvalues and eigenvectors of the matrix have to be calculated numerically at every interface and at every time step, which is computationally expensive;

- they do not satisfy in general an entropy inequality; as a consequence, an entropy-fix technique has to be added to the numerical scheme in order to capture the entropy solution in the presence of smooth transitions (see [14]);

- the positivity-preserving properties of the exact solutions of the problems are not satisfied in general by the numerical solutions.

The goal of this article is to design numerical methods based on a Roe linearization that overcome the first of these drawbacks of Roe methods. Moreover, some of the numerical schemes introduced here will also overcome the second and the third drawbacks.

For hyperbolic systems of conservation laws, it is well known that the use of incomplete Riemann solvers such as Rusanov, Lax-Friedrichs, HLL, etc. allows one to reduce the cpu time required by a Roe solver which resolves all the characteristic fields (see, for instance, 11]). Although when combined with piecewise constant approximation Roe solvers give in general a better resolution of the discontinuities than incomplete Riemann solvers, when combined with high order reconstructions the resolution may be indistinguishable. Therefore high order methods based on incomplete Riemann solvers may be more efficient than high order Roe methods. The idea here is to try to extend this strategy to nonconservative hyperbolic systems.

Even if all the numerical schemes considered here are based on a Roe linearization, the information concerning the eigenstructure of the intermediate matrices will not be used, in which case the corresponding numerical scheme is symmetric or nonupwind, or only partially used. In fact, the Roe matrices will be used here to compute in an easy manner the integrals through paths related to the definition of the nonconservative terms: when such a matrix is available, these integrals may 
be calculated by a simple matrix/vector product. In [4 a different approach to construct nonupwind schemes for nonconservative systems has been put forward, which is based on a reinterpretation of the PRICE-T method developed in [26] using the theoretical framework of path-conservative schemes. The main difference is that in their case, instead of using Roe matrices, the integrals through paths are computed numerically using Gaussian quadrature.

The organization of the paper is as follows: in Section 2 we recall some basic concepts. Section 3 is the core of the article. In the first paragraph, we introduce a family of numerical methods that generalizes the conservative Rusanov schemes. The Lax-Friedrichs method for nonconservative systems introduced and analyzed in [6] can be considered as a particular case. As their conservative counterpart, these methods satisfy a discrete entropy inequality and they are positivity-preserving. However, they are not well-balanced in general. In order to overcome this drawback, we present some well-balanced versions of these methods based on two different strategies: the first one is based on a modification of the identity matrix appearing in the definition of the methods; the second one consists of using the generalized hydrostatic reconstruction introduced in 9 which is an extension of the technique introduced in 1 for the numerical treatment of the source term in the shallow-water system.

Rusanov and Lax-Friedrichs schemes are very diffusive. The strategy followed here to reduce the numerical diffusion is inspired in the definition of the FORCE or GFORCE methods for conservative problems (see 25, 27]). In these methods, the numerical diffusion of the Lax-Friedrichs scheme is reduced by considering a conservative method whose flux is a convex linear combination of the Lax-Friedrichs and Lax-Wendroff fluxes. To follow such a strategy, first a generalization of LaxWendroff methods for nonconservative systems has to be defined. This is done in two different ways: while the first generalization is only second order for conservative problems, the second one is second order for general systems. Once the Lax-Friedrichs and the Lax-Wendroff methods have been generalized, we consider in Subsection 3.4 linear convex combinations of these methods. We thus obtain two different families of numerical schemes (depending on the choice of the first or the second order extension of the Lax-Wendroff scheme) which depend on a parameter $\omega \in[0,1]$. In particular, the choices $\omega=0,1,1 / 2,1 /(1+C F L)$ correspond to the extensions of Lax-Friedrichs, Lax-Wendroff, FORCE, and GFORCE numerical schemes, respectively.

In Section 4 the particular expression of these methods for systems of conservation laws with source terms and/or nonconservative products is obtained. The difficulty related to resonance is also discussed.

In Section 5 the numerical schemes introduced here are applied to the one and the two-layer shallow water systems and compared between them and with the usual Roe scheme. Together with the first order schemes presented here, we also consider their high order extensions based on the use of a reconstruction operator following the ideas given in [7, 20. As expected, the numerical results given by the first order numerical schemes introduced here are worse than those given by Roe methods, but when they are extended to higher order, the quality of the results is similar. Moreover, they are easier to implement and the numerical cost is dramatically reduced for problems in which the eigenvalues and eigenvectors are not explicitly known. 


\section{Preliminaries}

We consider one-dimensional P.D.E. systems of the form:

$$
W_{t}+\mathcal{A}(W) \cdot W_{x}=0, \quad x \in \mathbb{R}, t>0,
$$

where the unknown $W(x, t)$ takes values on an open convex set $\Omega$ of $\mathbb{R}^{N}$. The system is supposed to be strictly hyperbolic and the characteristic fields $R_{i}(W)$, $i=1, \ldots, N$, are supposed to be either genuinely nonlinear:

$$
\nabla \lambda_{i}(W) \cdot R_{i}(W) \neq 0, \quad \forall W \in \Omega,
$$

or linearly degenerate:

$$
\nabla \lambda_{i}(W) \cdot R_{i}(W)=0, \quad \forall W \in \Omega .
$$

Here, $\lambda_{1}(W), \ldots, \lambda_{N}(W)$ represent the eigenvalues of $\mathcal{A}(W)$ (in increasing order) and $R_{1}(W), \ldots, R_{N}(W)$ a set of associated eigenvectors.

Solutions of nonlinear hyperbolic systems are generally discontinuous. Due to the nondivergence form of the equations, the notion of solutions in the sense of distributions cannot be used. The theory introduced by Dal Maso, LeFloch, and Murat [10] is followed here to define weak solutions of (2.1). This theory allows one to define the nonconservative product $\mathcal{A}(W) W_{x}$ as a bounded measure provided that a family of Lipschitz continuous paths $\Phi:[0,1] \times \Omega \times \Omega$ is prescribed, which must satisfy certain natural regularity conditions; in particular,

$$
\Phi\left(0 ; W_{L}, W_{R}\right)=W_{L}, \quad \Phi\left(1 ; W_{L}, W_{R}\right)=W_{R}
$$

and

$$
\Phi(s ; W, W)=W .
$$

See [10] for details.

As occurs in the conservative case, not every discontinuity is admissible. Therefore, a concept of entropy solution has to be assumed. Here, we assume that there exists an entropy pair $(\eta, G)$, i.e. a pair of regular functions from $\Omega$ to $\mathbb{R}, \eta$ being convex, such that

$$
\nabla G(W)=\nabla \eta(W) \cdot \mathcal{A}(W), \quad \forall W \in \Omega .
$$

A weak solution is said to be an entropy solution if it satisfies the inequality

$$
\partial_{t} \eta(W)+\partial_{x} G(W) \leq 0
$$

in the distributions sense.

In order to discretize (2.1) we consider here path-conservative methods (see [20]) of the form:

$$
W_{i}^{n+1}=W_{i}^{n}-\frac{\Delta t}{\Delta x}\left(D_{i-1 / 2}^{+}+D_{i+1 / 2}^{-}\right)
$$

where $\Delta x$ and $\Delta t$ are, for simplicity, assumed to be constant; $W_{i}^{n}$ is the approximation provided by the numerical scheme of the cell average of the exact solution at the $i$-th cell, $I_{i}=\left[x_{i-1 / 2}, x_{i+1 / 2}\right]$ at the $n$-th time level $t^{n}=n \Delta t$, and

$$
D_{i+1 / 2}^{ \pm}=D^{ \pm}\left(W_{i}^{n}, \ldots, W_{i+1}^{n}\right)
$$

where $D^{-}$and $D^{+}$are two Lipschitz continuous functions from $\Omega^{2}$ to $\Omega$ satisfying:

$$
D^{ \pm}(W, W)=0, \quad \forall W \in \Omega,
$$


and for every pair of states $W_{L}, W_{R}$ :

$$
D^{-}\left(W_{L}, W_{R}\right)+D^{+}\left(W_{L}, W_{R}\right)=\int_{0}^{1} \mathcal{A}\left(\Phi\left(s ; W_{L}, W_{R}\right)\right) \frac{\partial \Phi}{\partial s}\left(s ; W_{L}, W_{R}\right) d s .
$$

The family of generalized Roe schemes introduced in 28 constitutes a particular case of path-conservative numerical methods. These schemes are based on the following general concept of a Roe linearization for (2.1): given a family of paths $\Phi$, a function $\mathcal{A}_{\Phi}: \Omega \times \Omega \mapsto \mathcal{M}_{N \times N}(\mathbb{R})$ is called a Roe linearization if it verifies the following properties:

- for any $W_{L}, W_{R} \in \Omega, \mathcal{A}_{\Phi}\left(W_{L}, W_{R}\right)$ has $N$ distinct real eigenvalues,

- for every $W \in \Omega$,

$$
\mathcal{A}_{\Phi}(W, W)=\mathcal{A}(W)
$$

- for any $W_{L}, W_{R} \in \Omega$,

$$
\mathcal{A}_{\Phi}\left(W_{L}, W_{R}\right) \cdot\left(W_{R}-W_{L}\right)=\int_{0}^{1} \mathcal{A}\left(\Phi\left(s ; W_{L}, W_{R}\right)\right) \frac{\partial \Phi}{\partial s}\left(s ; W_{L}, W_{R}\right) d s .
$$

Once a Roe linearization has been chosen, the corresponding Roe scheme is given by (2.4) together with:

$$
D_{i+1 / 2}^{ \pm}=\mathcal{A}_{i+1 / 2}^{ \pm} \cdot\left(W_{i+1}^{n}-W_{i}^{n}\right),
$$

where

$$
\mathcal{A}_{i+1 / 2}=\mathcal{A}_{\Phi}\left(W_{i}^{n}, W_{i+1}^{n}\right),
$$

and $\mathcal{A}_{i+1 / 2}^{ \pm}$denote its positive and negative parts, i.e.

$$
\mathcal{A}_{i+1 / 2}^{ \pm}=\mathcal{K}_{i+1 / 2} \mathcal{L}_{i+1 / 2}^{ \pm} \mathcal{K}_{i+1 / 2}^{-1}, \quad \mathcal{L}_{i+1 / 2}^{ \pm}=\left[\begin{array}{ccc}
\left(\lambda_{1}^{i+1 / 2}\right)^{ \pm} & & 0 \\
& \ddots & \\
0 & & \left(\lambda_{N}^{i+1 / 2}\right)^{ \pm}
\end{array}\right]
$$

$\lambda_{1}^{i+1 / 2}<\lambda_{2}^{i+1 / 2}<\cdots<\lambda_{N}^{i+1 / 2}$ being the eigenvalues of $\mathcal{A}_{i+1 / 2}$ and $\mathcal{K}_{i+1 / 2}$ an $N \times N$ matrix whose columns are the associated eigenvectors.

Well-balancing is related to the numerical approximation of steady state solutions. Notice that system (2.1) can only have nontrivial steady state solutions if it has at least one linearly degenerate field: if $W(x)$ is a smooth nontrivial stationary solution

$$
\mathcal{A}(W(x)) \cdot W^{\prime}(x)=0, \quad \forall x \in \mathbb{R},
$$

then 0 is an eigenvalue of $\mathcal{A}(W(x))$ and $W^{\prime}(x)$ an associated eigenvector for every $x$ such that $W^{\prime}(x) \neq 0$. Therefore, given an interval $J \subset \mathbb{R}$ such that $W^{\prime}(x) \neq 0$ for every $x$ in the interior of $J, x \in J \rightarrow W(x)$ is a parameterization of an arc of an integral curve of a characteristic field. Moreover, as the corresponding eigenvalue vanishes identically, the characteristic field has to be linearly degenerate.

Let us introduce the set $\Gamma$ of all the integral curves $\gamma$ of a linearly degenerate field of $\mathcal{A}(W)$ such that the corresponding eigenvalue vanishes on $\Gamma$. According to [21, we introduce the following definitions: 
Definition 2.1. Given a curve $\gamma \in \Gamma$, a numerical scheme for solving (2.1) is said to be exactly well-balanced for $\gamma$ if it solves exactly any smooth stationary solution $W$ such that

$$
W(x) \in \gamma, \quad \forall x .
$$

The scheme is said to be well-balanced of order $k$ for $\gamma$ if it solves up to order $k$ any smooth stationary solution satisfying (2.10). Finally, the scheme is said to be exactly well-balanced or well-balanced of order $k$ if these properties are satisfied for any curve of $\Gamma$.

Definition 2.1 generalizes the usual concept of well-balanced scheme for a system of balance laws (see [3]). It is a rather formal and geometrical definition, but it is well suited to the analysis of the well-balanced properties of path-conservative numerical schemes as these properties are strongly connected to the relationship between the paths and the set of curves $\Gamma$; see 20. For instance, the following general result can be easily shown for Roe methods (see [21):

Proposition 2.2. Let $\gamma$ be a curve in $\Gamma$. Let $\mathcal{A}_{\Phi}$ be a Roe linearization related to a family of paths $\Phi$ satisfying that given two states $W_{L}$ and $W_{R}$ belonging to $\gamma$, the path $\Phi\left(\cdot ; W_{L}, W_{R}\right)$ is a parameterization of the arc of $\gamma$ linking the states. Then, the corresponding Roe scheme is exactly well-balanced for $\gamma$.

The proof of this proposition is straightforward: on the one hand, it can be easily deduced from (2.7) that, given two states $W_{L}$ and $W_{R}$ belonging to $\gamma$, the following equality holds:

$$
\mathcal{A}_{\Phi}\left(W_{L}, W_{R}\right) \cdot\left(W_{R}-W_{L}\right)=0 .
$$

The proof is easily concluded by using the definitions of the positive and negative parts, and the form (2.4) of the scheme.

\section{NuMERICAL SCHEMES}

The $D^{ \pm}$functions of the new families of path-conservative numerical schemes introduced here will be of the form:

$$
D_{i+1 / 2}^{ \pm}=\widehat{\mathcal{A}}_{\Phi}^{ \pm}\left(W_{i}^{n}, W_{i+1}^{n}\right) \cdot\left(W_{i+1}^{n}-W_{i}^{n}\right),
$$

where

$$
\mathcal{A}_{\Phi}\left(W_{L}, W_{R}\right)=\widehat{\mathcal{A}}_{\Phi}^{+}\left(W_{L}, W_{R}\right)+\widehat{\mathcal{A}}_{\Phi}^{-}\left(W_{L}, W_{R}\right)
$$

represents an arbitrary decomposition of the Roe linearization. In particular, the decomposition

$$
\widehat{\mathcal{A}}_{\Phi}^{ \pm}\left(W_{L}, W_{R}\right)=\mathcal{A}_{\Phi}^{ \pm}\left(W_{L}, W_{R}\right),
$$

where $\mathcal{A}_{\Phi}^{ \pm}\left(W_{L}, W_{R}\right)$ are the positive and negative parts of the matrix corresponds to the Roe method.

Clearly, a numerical scheme of the form (2.4), (3.1) based on a decomposition (3.2) of a Roe linearization $\mathcal{A}_{\Phi}$ is well-balanced for a curve $\gamma \in \Gamma$ if, and only if, given two states $W_{L}$ and $W_{R}$ belonging to $\gamma$, the following equalities hold:

$$
\widehat{\mathcal{A}}_{\Phi}^{ \pm}\left(W_{L}, W_{R}\right) \cdot\left(W_{R}-W_{L}\right)=0 .
$$


3.1. Rusanov methods. Let us consider first the numerical schemes (2.4), (3.1) based on the decomposition (3.2) given by:

$$
\begin{aligned}
\widehat{\mathcal{A}}_{\Phi}^{+}\left(W_{L}, W_{R}\right) & =\frac{1}{2}\left[\alpha\left(W_{L}, W_{R}\right) I d+\mathcal{A}_{\Phi}\left(W_{L}, W_{R}\right)\right], \\
\widehat{\mathcal{A}}_{\Phi}^{-}\left(W_{L}, W_{R}\right) & =\frac{1}{2}\left[-\alpha\left(W_{L}, W_{R}\right) I d+\mathcal{A}_{\Phi}\left(W_{L}, W_{R}\right)\right],
\end{aligned}
$$

where $\alpha\left(W_{L}, W_{R}\right)$ is a positive number such that:

$$
\left|\lambda_{i}\left(W_{L}, W_{R}\right)\right| \leq \alpha\left(W_{L}, W_{R}\right), \quad i=1, \ldots, N .
$$

Here, $\lambda_{i}\left(W_{L}, W_{R}\right), i=1, \ldots, N$ represent the eigenvalues of the Roe matrix. Clearly, the eigenvalues of $\widehat{\mathcal{A}}_{\Phi}^{+}\left(W_{L}, W_{R}\right)$ and $\widehat{\mathcal{A}}_{\Phi}^{-}\left(W_{L}, W_{R}\right)$ are, respectively, positive and negative. We remark that this method only requires a partial knowledge of the eigenstructure: at every interface only the maximum eigenvalue (or an estimate) has to be known.

It can be easily verified that, for conservative problems, this family of schemes reduces to the conservative methods based on Rusanov numerical fluxes. Schemes of this family are first order accurate and linearly stable under the usual CFL condition:

$$
\frac{\Delta t}{\Delta x} \max \left\{\left|\lambda_{j}\left(W_{i}^{n}, W_{i+1}^{n}\right)\right|: j=1, \ldots, N\right\}=\mathrm{CFL}, \quad \forall i,
$$

where CFL $\in(0,1]$. Moreover, they have good entropy-satisfying and positivitypreserving properties: under some hypotheses on $\Phi$ (see Appendix A), the following results hold:

Proposition 3.1. Let us assume that $\alpha\left(W_{L}, W_{R}\right)$ satisfies:

$$
\max \left\{\left|\lambda_{j}\left(W_{k}\right)\right|: j=1, \ldots, N\right\} \leq \alpha\left(W_{L}, W_{R}\right), \quad k=0, \ldots, N,
$$

where $W_{0}, \ldots, W_{N}$ are the intermediate states appearing in the self-similar entropy solution $V\left(x / t ; W_{L}, W_{R}\right)$ of the Riemann problem corresponding to $W_{L}, W_{R}$. Then, under the $C F L$ assumption:

$$
\frac{\Delta t}{\Delta x} \alpha\left(W_{i}^{n}, W_{i+1}^{n}\right) \leq \frac{1}{2}, \quad \forall i
$$

there exists a consistent numerical entropy flux function $\widehat{G}\left(W_{L}, W_{R}\right)$ such that the following entropy inequality is satisfied:

$$
\eta\left(W_{i}^{n+1}\right)-\eta\left(W_{i}^{n}\right)+\frac{\Delta t}{\Delta x}\left(\widehat{G}\left(W_{i}^{n}, W_{i+1}^{n}\right)-\widehat{G}\left(W_{i-1}^{n}, W_{i}^{n}\right)\right) \leq 0 .
$$

Proposition 3.2. Under the hypothesis of Proposition 3.1, let us suppose that the Riemann problem has the following positivity-preserving property: there exists $j$ such that:

$$
w_{j}^{L}, w_{j}^{R} \geq 0 \Longrightarrow V_{j}\left(v ; W_{L}, W_{R}\right) \geq 0, \quad \forall v,
$$

where $w_{j}^{L}, w_{j}^{R}, V_{j}\left(\cdot ; W_{L}, W_{R}\right)$ represent, respectively, the $j$-th component of $W_{L}$, $W_{R}$, and $V\left(\cdot ; W_{L}, W_{R}\right)$. Then, the Rusanov method based on the decomposition (3.5), (3.6) is also positivity-preserving in the following sense:

$$
w_{i, j}^{0} \geq 0, \quad \forall i \Longrightarrow w_{i, j}^{n} \geq 0, \quad \forall i, n,
$$

where $w_{i, j}^{n}$ represents the $j$-th component of $W_{i}^{n}$. 
These results are proved in Appendix A. The proof is based on the interpretation of the methods as approximate Riemann solvers.

Notice that, if $\Delta x$ and $\Delta t$ satisfy (3.7), a possible choice for $\alpha$ is given by:

$$
\alpha\left(W_{i}^{n}, W_{i+1}^{n}\right)=\frac{\Delta x}{\Delta t}, \quad \forall i .
$$

For conservative problems, the corresponding numerical scheme reduces to the LaxFriedrichs method. In this case no local information is used in the expression of the numerical scheme. Only an estimate of the maximum speed in the whole of the domain at any given time level is needed in order to impose the CFL condition.

By using the Roe property (2.7), this generalized Lax-Friedrichs method can also be written in the form (2.4) with:

$$
D_{i+1 / 2}^{ \pm}=\int_{0}^{1} \widehat{A}^{ \pm}\left(\Phi\left(s ; W_{i}^{n}, W_{i+1}^{n}\right)\right) \frac{\partial \Phi}{\partial s}\left(s ; W_{i}^{n}, W_{i+1}^{n}\right) d s,
$$

where

$$
\widehat{A}^{ \pm}(W)=\frac{1}{2}\left( \pm \frac{\Delta x}{\Delta t} I d+\mathcal{A}(W)\right) .
$$

The Lax-Friedrichs method written in this form has been introduced and analyzed in 6]. From equation (3.13), it is clear that the method is independent of the particular choice of Roe linearization. Nevertheless, its practical implementation is easier when a Roe linearization is available.

3.2. Well-balanced Rusanov schemes. Let us consider in this section a Roe linearization based on a family $\Phi$ satisfying the hypotheses of Proposition 2.2. The corresponding Roe scheme is thus well-balanced for $\gamma$.

In general, Rusanov schemes based on the decomposition (3.5), (3.6) are not well-balanced for $\gamma$. In effect, given $W_{L}, W_{R} \in \gamma$, we have the equality:

$$
\widehat{\mathcal{A}}_{\Phi}^{ \pm}\left(W_{L}, W_{R}\right)\left(W_{R}-W_{L}\right)= \pm \frac{1}{2} \alpha\left(W_{L}, W_{R}\right)\left(W_{R}-W_{L}\right) \neq 0 .
$$

In this section, we introduce two different strategies to make the numerical scheme well-balanced.

3.2.1. Modification of the identity matrix. Observe that, given $W_{L}, W_{R} \in \gamma$, equation (2.11) is satisfied and thus 0 is an eigenvalue of $\mathcal{A}_{\Phi}\left(W_{L}, W_{R}\right)$ and $W_{R}-W_{L}$ is an associated eigenvector. Let us consider the following decomposition:

$$
\begin{aligned}
\widehat{\mathcal{A}}_{\Phi}^{+}\left(W_{L}, W_{R}\right) & =\frac{1}{2}\left[\alpha\left(W_{L}, W_{R}\right) \widehat{I}\left(W_{L}, W_{R}\right)+\mathcal{A}_{\Phi}\left(W_{L}, W_{R}\right)\right], \\
\widehat{\mathcal{A}}_{\Phi}^{-}\left(W_{L}, W_{R}\right) & =\frac{1}{2}\left[-\alpha\left(W_{L}, W_{R}\right) \widehat{I}\left(W_{L}, W_{R}\right)+\mathcal{A}_{\Phi}\left(W_{L}, W_{R}\right)\right],
\end{aligned}
$$

where $\widehat{I}\left(W_{L}, W_{R}\right)$ is:

$$
\widehat{I}\left(W_{L}, W_{R}\right)=K\left(W_{L}, W_{R}\right) \cdot \widehat{I d} \cdot K\left(W_{L}, W_{R}\right)^{-1} .
$$

Here $K\left(W_{L}, W_{R}\right)$ is a matrix whose columns are eigenvectors of $\mathcal{A}_{\Phi}\left(W_{L}, W_{R}\right)$, and $\widehat{I d}$ is the diagonal matrix whose $i$-th coefficient is 1 if $\lambda_{i}\left(W_{L}, W_{R}\right) \neq 0$, or 0 if $\lambda_{i}\left(W_{L}, W_{R}\right)=0$.

Notice that, if the states $W_{L}$ and $W_{R}$ belong to $\gamma, W_{R}-W_{L}$ is an eigenvector associated to 0 for both the matrices $\mathcal{A}_{\Phi}\left(W_{L}, W_{R}\right)$ and $\widehat{I}\left(W_{L}, W_{R}\right)$. Therefore, (3.4) is satisfied and the numerical scheme is exactly well-balanced for $\gamma$. 
Again, the choice (3.12) provides a well-balanced version of the generalized LaxFriedrichs scheme.

This well-balanced version of the Rusanov and Lax-Friedrichs methods is also first order accurate and linearly stable under the usual CFL condition. Unfortunately, the proofs of Propositions 3.1 and 3.2 cannot be adapted in general to this modified scheme: the entropy-satisfying and positivity-preserving properties of the scheme may fail for the modified version.

Notice that in this well-balanced version of the scheme, at least the eigenvector associated with the null eigenvalue of the intermediate matrices has to be known.

3.2.2. Generalized hydrostatic reconstruction. In this paragraph we follow the strategy designed in [9] (which is a generalization of that introduced in [1] for the shallow water system) to modify any path-conservative numerical scheme (2.4) in order to be well-balanced for all the curves of a subset $\Gamma_{0}$ of $\Gamma$.

Let us assume that there exists a family of Lipschitz-continuous curves:

$$
\widetilde{\Gamma}=\left\{C_{W}, \quad W \in \Omega\right\}
$$

satisfying the following properties:

(P1) $W \in C_{W}$ for every $W \in \Omega$.

(P2) If $W$ belongs to a curve $\gamma \in \Gamma_{0}$, then

$$
C_{W}=\gamma .
$$

(P3) Given two arbitrary states $W_{0}$ and $W_{1}$ in $\Omega$, it is possible to choose in a continuous way two states $W_{1 / 2}^{-} \in C_{W_{0}}$ and $W_{1 / 2}^{+} \in C_{W_{1}}$ such that

$$
W_{1 / 2}^{-}=W_{1 / 2}^{+}
$$

whenever $W_{0}$ and $W_{1}$ belong to the same curve $\gamma \in \Gamma_{0}$.

The following modification of the numerical scheme (2.4) is then considered:

$$
\begin{aligned}
W_{i}^{n+1}= & W_{i}^{n}-\frac{\Delta t}{\Delta x}\left(D^{+}\left(W_{i-1 / 2}^{-}, W_{i-1 / 2}^{+}\right)+D^{-}\left(W_{i+1 / 2}^{-}, W_{i+1 / 2}^{+}\right)\right. \\
& \left.+\int_{0}^{1} \mathcal{A}\left(Q_{i-1 / 2}^{+}(s)\right) \frac{d Q_{i-1 / 2}^{+}}{d s} d s+\int_{0}^{1} \mathcal{A}\left(Q_{i+1 / 2}^{-}(s)\right) \frac{d Q_{i+1 / 2}^{-}}{d s} d s\right),
\end{aligned}
$$

where $W_{i+1 / 2}^{ \pm}$represent the reconstructed states obtained by applying (P3) to $W_{i}^{n}$ and $W_{i+1}^{n}$, and

$$
\begin{aligned}
& s \in[0,1] \quad \mapsto Q_{i+1 / 2}^{-}(s), \\
& s \in[0,1] \quad \mapsto Q_{i+1 / 2}^{+}(s),
\end{aligned}
$$

are, respectively, parameterizations of the arcs of the curves $C_{W_{i}^{n}}$ and $C_{W_{i+1}^{n}}$, linking the pairs of states $\left(W_{i}^{n}, W_{i+1 / 2}^{-}\right)$and $\left(W_{i+1 / 2}^{+}, W_{i+1}^{n}\right)$. The numerical scheme (3.19) is path-conservative, consistent and well-balanced for every curve $\gamma \in \Gamma$.

The entropy-satisfying and positivity-preserving properties of the chosen pathconservative scheme may also be satisfied by its modification (3.19) if the generalized hydrostatic reconstruction satisfies some properties. We give hereafter two general results which are satisfied for the particular case of the hydrostatic reconstruction introduced in [1] for the shallow water system with source terms. In fact, their proofs follow closely those of the corresponding results given in 3 . 
Proposition 3.3. Let us suppose that the numerical scheme (2.4) satisfies the following semi-discrete entropy inequality by interfaces: there exists a consistent numerical flux entropy such that

$$
\begin{aligned}
& G\left(W_{1}\right)+\nabla \eta\left(W_{1}\right) \cdot D^{-}\left(W_{0}, W_{1}\right) \leq \widehat{G}\left(W_{0}, W_{1}\right), \\
& G\left(W_{0}\right)+\nabla \eta\left(W_{0}\right) \cdot D^{+}\left(W_{0}, W_{1}\right) \geq \widehat{G}\left(W_{0}, W_{1}\right)
\end{aligned}
$$

hold for any pair of states $W_{0}$ and $W_{1}$. Let us also suppose that the reconstructed states satisfy the inequalities:

$$
\begin{gathered}
G\left(W_{1 / 2}^{-}\right)-G\left(W_{0}\right)+\left(\nabla \eta\left(W_{1 / 2}^{-}\right)-\nabla \eta\left(W_{0}\right)\right) \cdot D^{+}\left(W_{1 / 2}^{-}, W_{1 / 2}^{+}\right) \\
\leq \nabla \eta\left(W_{0}\right) \cdot \int_{0}^{1} \mathcal{A}\left(Q_{1 / 2}^{-}(s)\right) \frac{d Q_{1 / 2}^{-}}{d s} d s, \\
G\left(W_{1 / 2}^{+}\right)-G\left(W_{1}\right)+\left(\nabla \eta\left(W_{1 / 2}^{+}\right)-\nabla \eta\left(W_{1}\right)\right) \cdot D^{-}\left(W_{1 / 2}^{-}, W_{1 / 2}^{+}\right) \\
\geq \nabla \eta\left(W_{1}\right) \cdot \int_{0}^{1} \mathcal{A}\left(Q_{1 / 2}^{+}(s)\right) \frac{d Q_{1 / 2}^{+}}{d s} d s .
\end{gathered}
$$

Then, the numerical scheme (3.19) also satisfies a semi-discrete entropy inequality by interfaces for the consistent numerical flux function:

$$
\widetilde{G}\left(W_{0}, W_{1}\right)=\widehat{G}\left(W_{1 / 2}^{-}, W_{1 / 2}^{+}\right) .
$$

Proposition 3.4. Let us suppose that the numerical scheme (2.4) is positivitypreserving in the sense of Proposition 3.2. Let us also suppose that, given two states

$$
W_{k}=\left[w_{k, 1}, \ldots, w_{k, N}\right]^{T}, \quad k=0,1,
$$

the reconstructed states

$$
W_{1 / 2}^{ \pm}=\left[w_{1 / 2,1}^{ \pm}, \ldots, w_{1 / 2, N}^{ \pm}\right]^{T}
$$

are such that

$$
w_{0, j} \geq w_{1 / 2, j}^{-}, \quad w_{1, j} \geq w_{1 / 2, j}^{+}
$$

and

$$
\int_{0}^{1} \sum_{l=1}^{N} a_{j, l}\left(Q_{1 / 2}^{-}(s)\right) \frac{d q_{1 / 2, l}^{-}}{d s} d s+\int_{0}^{1} \sum_{l=1}^{N} a_{j, l}\left(Q_{1 / 2}^{+}(s)\right) \frac{d q_{1 / 2, l}^{+}}{d s} d s \leq 0,
$$

where $a_{j, l}(W)$ represents the $(j, l)$-entry of the matrix $\mathcal{A}(W)$, and

$$
s \mapsto Q_{1 / 2}^{ \pm}(s)=\left[q_{1 / 2,1}^{ \pm}(s), \ldots, q_{1 / 2, N}^{ \pm}(s)\right]^{T}
$$

are some parameterizations of the arcs of $C_{W_{0}}$ and $C_{W_{1}}$, linking the pairs of states $\left(W_{0}, W_{1 / 2}^{-}\right)$and $\left(W_{1 / 2}^{+}, W_{1}\right)$. Then the numerical scheme (3.19) is also positivitypreserving. 
3.3. Generalized Lax-Wendroff schemes. We consider now the numerical scheme defined by the following decomposition:

$$
\begin{aligned}
\widehat{\mathcal{A}}_{\Phi}^{+}\left(W_{L}, W_{R}\right) & =\frac{1}{2}\left[\mathcal{A}_{\Phi}\left(W_{L}, W_{R}\right)+\frac{\Delta t}{\Delta x} \mathcal{A}_{\Phi}^{2}\left(W_{L}, W_{R}\right)\right] \\
\widehat{\mathcal{A}}_{\Phi}^{-}\left(W_{L}, W_{R}\right) & =\frac{1}{2}\left[\mathcal{A}_{\Phi}\left(W_{L}, W_{R}\right)-\frac{\Delta t}{\Delta x} \mathcal{A}_{\Phi}^{2}\left(W_{L}, W_{R}\right)\right] .
\end{aligned}
$$

For linear or conservative problems these schemes reduce to conservative methods based on Lax-Wendroff numerical fluxes, and they are second order accurate in these cases. However, in the presence of nonconservative terms they are only first order accurate. In effect, a Taylor series expansion allows us to prove the following development for a smooth solution of (2.1) with bounded derivatives:

$$
\begin{aligned}
& W(x, t+\Delta t)=W(x, t)-\Delta t \mathcal{A}(W) \cdot W_{x}+\frac{\Delta t^{2}}{2} \partial_{x}\left(\mathcal{A}(W)^{2} \cdot W_{x}\right) \\
& +\frac{\Delta t^{2}}{2}\left(D \mathcal{A}(W)\left[\mathcal{A}(W) \cdot W_{x}, W_{x}\right]-D \mathcal{A}(W)\left[W_{x}, \mathcal{A}(W) \cdot W_{x}\right]\right)+O\left(\Delta t^{3}\right),
\end{aligned}
$$

where the dependence on $x, t$ of the terms involving derivatives has been dropped for simplicity, and the following notation has been used: given two vectors

$$
U=\left[u_{1}, \ldots, u_{N}\right]^{T}, \quad V=\left[v_{1}, \ldots, v_{N}\right]^{T},
$$

$D \mathcal{A}(W)[U, V]$ represents the derivative of $\mathcal{A}(W)$ in the direction of the vector $U$ applied to the vector $W$, i.e.

$$
D \mathcal{A}(W)[U, V]=\left(\sum_{l=1}^{N} u_{l} \partial_{w_{l}} \mathcal{A}(W)\right) \cdot V .
$$

Here, $\partial_{w_{l}} \mathcal{A}(W)$ is the $N \times N$ matrix whose $(i, j)$ entry is

$$
\partial_{w_{l}} a_{i, j}(W) \text {. }
$$

Let us consider the numerical scheme given by

$$
\begin{aligned}
& D^{+}\left(W_{L}, W_{R}\right)=\frac{1}{2}\left[\mathcal{A}_{\Phi}\left(W_{L}, W_{R}\right) \cdot\left(W_{R}-W_{L}\right)+\frac{\Delta t}{\Delta x} \mathcal{A}_{\Phi}^{2}\left(W_{L}, W_{R}\right) \cdot\left(W_{R}-W_{L}\right)\right. \\
& -\frac{\Delta t}{2 \Delta x}\left(D \mathcal{A}\left(W_{R}\right)\left[\mathcal{A}_{\Phi}\left(W_{L}, W_{R}\right) \cdot\left(W_{R}-W_{L}\right), W_{R}-W_{L}\right]\right. \\
& \left.\left.-D \mathcal{A}\left(W_{R}\right)\left[W_{R}-W_{L}, \mathcal{A}_{\Phi}\left(W_{L}, W_{R}\right) \cdot\left(W_{R}-W_{L}\right)\right]\right)\right] \\
& D^{-}\left(W_{L}, W_{R}\right) \frac{1}{2}\left[\mathcal{A}_{\Phi}\left(W_{L}, W_{R}\right) \cdot\left(W_{R}-W_{L}\right)-\frac{\Delta t}{\Delta x} \mathcal{A}_{\Phi}^{2}\left(W_{L}, W_{R}\right) \cdot\left(W_{R}-W_{L}\right)\right. \\
& -\frac{\Delta t}{2 \Delta x}\left(D \mathcal{A}\left(W_{L}\right)\left[\mathcal{A}_{\Phi}\left(W_{L}, W_{R}\right) \cdot\left(W_{R}-W_{L}\right), W_{R}-W_{L}\right]\right. \\
& \left.\left.-D \mathcal{A}\left(W_{L}\right)\left[W_{R}-W_{L}, \mathcal{A}_{\Phi}\left(W_{L}, W_{R}\right) \cdot\left(W_{R}-W_{L}\right)\right]\right)\right] .
\end{aligned}
$$

This scheme is second order accurate for general problems.

Both the first and the second order extensions of the Lax-Wendroff scheme introduced in this section preserve the well-balanced properties of the Roe scheme corresponding to the chosen linearization: let us suppose again that the hypothesis 
of Proposition 2.2 is satisfied. Then, 2.11) is satisfied and it can be easily verified that:

$$
\widehat{\mathcal{A}}_{\Phi}^{ \pm}\left(W_{L}, W_{R}\right) \cdot\left(W_{R}-W_{L}\right)=0, D^{ \pm}\left(W_{L}, W_{R}\right)=0,
$$

for $\widehat{\mathcal{A}}_{\Phi}^{ \pm}\left(W_{L}, W_{R}\right)$ given by (3.24)-3.25) and $D^{ \pm}\left(W_{L}, W_{R}\right)$ given by (3.26)-3.27).

The schemes introduced in this paragraph are expected to produce oscillations near the discontinuities, as happens for conservative systems. The idea here is to construct numerical schemes which are a combination of the generalized LaxFriedrichs and Lax-Wendroff schemes, in order to reduce the numerical viscosity of the first one without losing its good stability properties.

\subsection{Convex linear combinations of Lax-Friedrichs and Lax-Wendroff}

schemes. In this paragraph, we consider decompositions which are obtained by means of a convex linear combination of those corresponding to Lax-Wendroff and Lax-Friedrichs schemes. First, we consider:

$$
\widehat{\mathcal{A}}_{\Phi}^{ \pm}\left(W_{L}, W_{R}\right)=(1-\omega) \widehat{\mathcal{A}}_{\Phi, L F}^{ \pm}\left(W_{L}, W_{R}\right)+\omega \widehat{\mathcal{A}}_{\Phi, L W}^{ \pm}\left(W_{L}, W_{R}\right),
$$

where $\omega$ is a fixed parameter in $[0,1], \widehat{\mathcal{A}}_{\Phi, L F}^{ \pm}\left(W_{L}, W_{R}\right)$ are given by (3.5)-(3.6) with the choice (3.12), and $\widehat{\mathcal{A}}_{\Phi, L W}^{ \pm}\left(W_{L}, W_{R}\right)$ are given by (3.24)-(3.25). It is interesting to note that the eigenvalues of $\widehat{\mathcal{A}}_{\Phi}^{+}\left(W_{L}, W_{R}\right)$ (resp. $\widehat{\mathcal{A}}_{\Phi}^{-}\left(W_{L}, W_{R}\right)$ ) are positive (resp. negative) if

$$
\omega \leq \frac{1}{1+\mathrm{CFL}}
$$

given that this is precisely the region of all monotone schemes allowed by the convex average for linear scalar problems. In that case, the particular choice $\omega=1 /(1+c)$ corresponds to the Godunov upwind scheme.

We will only consider here the choices $\omega=0,1,1 / 2,1 /(1+\mathrm{CFL})$ that correspond to the particular cases of the generalized Lax-Friedrichs, Lax-Wendroff, FORCE, and GFORCE numerical schemes.

In general, the scheme based on the decomposition (3.28) is not well-balanced for $\gamma$ under the hypotheses of Proposition 2.2. A well-balanced numerical scheme can again be recovered if the following decomposition is considered:

$$
\widehat{\mathcal{A}}_{\Phi}^{ \pm}\left(W_{L}, W_{R}\right)=(1-\omega) \widehat{\mathcal{A}}_{\Phi, W B L F}^{ \pm}\left(W_{L}, W_{R}\right)+\omega \widehat{\mathcal{A}}_{\Phi, L W}^{ \pm}\left(W_{L}, W_{R}\right),
$$

where now $\widehat{\mathcal{A}}_{\Phi, W B L F}^{ \pm}\left(W_{L}, W_{R}\right)$ are given by (3.14)-(3.15) together with the choice (3.12).

Finally, we also consider the following combinations of the Lax-Friedrichs scheme and the second order extension of the Lax-Wendroff scheme:

$$
D^{ \pm}\left(W_{L}, W_{R}\right)=(1-\omega) \widehat{\mathcal{A}}_{\Phi, L F}^{ \pm}\left(W_{L}, W_{R}\right) \cdot\left(W_{R}-W_{L}\right)+\omega D_{L W}^{ \pm}\left(W_{L}, W_{R}\right),
$$

where $D_{L W}^{ \pm}$are given by (3.26)-(3.27), or its well-balanced version, in which $\widehat{\mathcal{A}}_{\Phi, L F}^{ \pm}\left(W_{L}, W_{R}\right)$ are replaced by $\widehat{\mathcal{A}}_{\Phi, W B L F}^{ \pm}\left(W_{L}, W_{R}\right)$.

\section{Systems of CONSERVATION LAWS With SOURCE TERMS AND NONCONSERVATIVE PRODUCTS}

We consider in this section PDE systems of the form:

$$
w_{t}+F(w)_{x}+\mathcal{B}(w) \cdot w_{x}=S(w) \sigma_{x},
$$


where the unknown $w(x, t)$ takes values on an open convex set $\mathcal{O}$ of $\mathbb{R}^{N} ; F$ is a regular function from $\mathcal{O}$ to $\mathbb{R}^{N}$, whose Jacobian matrix will be denoted by $\mathcal{J}(w)$ :

$$
\partial(w)=\frac{\partial F}{\partial w}(w)
$$

$\mathcal{B}$ is a regular matrix function from $\mathcal{O}$ to $\mathcal{M}_{N \times N}(\mathbb{R}) ; S$ is a function from $\mathcal{O}$ to $\mathbb{R}^{N}$; and $\sigma(x)$ is a known function from $\mathbb{R}$ to $\mathbb{R}$.

By adding to (4.1) the equation

$$
\sigma_{t}=0
$$

the system can be rewritten in the form (2.1):

$$
W_{t}+\widetilde{\mathcal{A}}(W) \cdot W_{x}=0,
$$

where $W$ is the augmented vector

$$
W=\left[\begin{array}{c}
w \\
\sigma
\end{array}\right] \in \Omega=\mathcal{O} \times \mathbb{R}
$$

and $\widetilde{\mathcal{A}}(W)$ is the $(N+1) \times(N+1)$ matrix whose block structure is given by

$$
\widetilde{\mathcal{A}}(W)=\left[\begin{array}{c|c}
\mathcal{A}(w) & -S(w) \\
\hline 0 & 0
\end{array}\right] .
$$

Here,

$$
\mathcal{A}(w)=\mathcal{J}(w)+\mathcal{B}(w)
$$

which is assumed to have $N$ real distinct eigenvalues

$$
\lambda_{1}(w)<\cdots<\lambda_{N}(w),
$$

and associated eigenvectors $r_{j}(w), j=1, \ldots, N$. If the eigenvalues $\lambda_{1}(w), \ldots, \lambda_{N}(w)$ of $\mathcal{A}(w)$ do not vanish, (4.3) is a strictly hyperbolic system: $\widetilde{\mathcal{A}}(W)$ has $N+1$ distinct real eigenvalues

$$
\lambda_{1}(w), \ldots, \lambda_{N}(w), 0,
$$

with associated eigenvectors

$$
R_{1}(W), \ldots, R_{N+1}(W),
$$

given by

$$
R_{i}(W)=\left[\begin{array}{c}
r_{i}(w) \\
0
\end{array}\right], i=1, \ldots, N ; \quad R_{N+1}(W)=\left[\begin{array}{c}
\mathcal{A}(w)^{-1} \cdot S(w) \\
1
\end{array}\right] .
$$

Clearly, the $(N+1)$-th field is linearly degenerate and, for the sake of simplicity, we assume that it is the only one, the others being genuinely nonlinear. In this case, the set $\Gamma$ is composed of all the interval curves of the $(N+1)$-th field, which are the integral curves of the o.d.e. system:

$$
\frac{d w}{d s}=\mathcal{A}(w)^{-1} \cdot S(w)
$$

In order to construct Roe linearizations of (4.3), first a family of paths $\widetilde{\Phi}$ in $\Omega$ has to be chosen. We will use the notation

$$
W_{L}=\left[\begin{array}{c}
w_{L} \\
\sigma_{L}
\end{array}\right], \quad W_{R}=\left[\begin{array}{c}
w_{R} \\
\sigma_{R}
\end{array}\right]
$$


for the states, and

$$
\widetilde{\Phi}\left(s ; W_{L}, W_{R}\right)=\left[\begin{array}{c}
\Phi\left(s ; W_{L}, W_{R}\right) \\
\Phi_{N+1}\left(s ; W_{L}, W_{R}\right)
\end{array}\right],
$$

where

$$
\Phi\left(s ; W_{L}, W_{R}\right)=\left[\begin{array}{c}
\Phi_{1}\left(s ; W_{L}, W_{R}\right) \\
\vdots \\
\Phi_{N}\left(s ; W_{L}, W_{R}\right)
\end{array}\right],
$$

for the paths connecting the states.

As in [21, we consider families of paths $\widetilde{\Phi}$ which are an extension to $\Omega$ of a family of paths in $\mathcal{O}$; i.e., we assume that there exists a family of paths $\phi:[0,1] \times \mathcal{O} \times \mathcal{O} \rightarrow \mathcal{O}$ such that, if $W_{L}$ and $W_{R}$ are two states such that $\sigma_{L}=\sigma_{R}=\bar{\sigma}$, then:

$$
\widetilde{\Phi}\left(s ; W_{L}, W_{R}\right)=\left[\begin{array}{c}
\phi\left(s ; w_{L}, w_{R}\right) \\
\bar{\sigma}
\end{array}\right], \quad \forall s \in[0,1] .
$$

This requirement is natural, as it ensures that for constant $\sigma$, the numerical schemes designed for (4.1) will reduce to $\phi$-conservative numerical schemes for the homogeneous problem:

$$
w_{t}+F(w)_{x}+\mathcal{B}(w) \cdot w_{x}=0 .
$$

Following [21, we consider Roe linearizations $\widetilde{\mathcal{A}}_{\widetilde{\Phi}}\left(W_{L}, W_{R}\right)$ of the form:

$$
\widetilde{\mathcal{A}}_{\widetilde{\Phi}}\left(W_{L}, W_{R}\right)=\left[\begin{array}{c|c}
\mathcal{A}_{\Phi}\left(W_{L}, W_{R}\right) & -S_{\widetilde{\Phi}}\left(W_{L}, W_{R}\right) \\
\hline 0 & 0
\end{array},\right.
$$

where

$$
\mathcal{A}_{\Phi}\left(W_{L}, W_{R}\right)=\mathcal{J}\left(w_{L}, w_{R}\right)+\mathcal{B}_{\Phi}\left(W_{L}, W_{R}\right) .
$$

Here, $\mathcal{J}\left(w_{L}, w_{R}\right)$ is a Roe linearization of the Jacobian of the flux $F$ in the usual sense:

$$
\mathcal{J}\left(w_{L}, w_{R}\right) \cdot\left(w_{R}-w_{L}\right)=F\left(w_{R}\right)-F\left(w_{L}\right) ;
$$

$\mathcal{B}_{\Phi}\left(W_{L}, W_{R}\right)$ is a matrix satisfying:

$$
\mathcal{B}_{\Phi}\left(W_{L}, W_{R}\right) \cdot\left(w_{R}-w_{L}\right)=\int_{0}^{1} \mathcal{B}\left(\Phi\left(s ; W_{L}, W_{R}\right)\right) \frac{\partial \Phi}{\partial s}\left(s ; W_{L}, W_{R}\right) d s
$$

and $S_{\widetilde{\Phi}}\left(W_{L}, W_{R}\right)$ is a vector satisfying:

$$
S_{\widetilde{\Phi}}\left(W_{L}, W_{R}\right)\left(\sigma_{R}-\sigma_{L}\right)=\int_{0}^{1} S\left(\Phi\left(s ; W_{L}, W_{R}\right)\right) \frac{\partial \Phi_{N+1}}{\partial s}\left(s ; W_{L}, W_{R}\right) d s .
$$

It can be easily shown that, if (4.8)-(4.10) are fulfilled, then the matrix defined by (4.6)-(4.7) satisfies (2.6) and (2.7) for the family of paths $\widetilde{\Phi}$. It is thus a Roe linearization provided that it has $N+1$ different real eigenvalues.

The non-well-balanced numerical versions of the schemes introduced in Section 3.4 are useless in this case, as they do not preserve equation (4.2), which is an obvious requirement. Let us thus apply their well-balanced versions. 
4.1. Modification of the identity matrix. Some algebraical calculations allow us to show that the matrix $\widehat{I}\left(W_{L}, W_{R}\right)$ given by (3.16) has, in this case, the following block structure:

$$
\widehat{I}\left(W_{L}, W_{R}\right)=\left[\begin{array}{c|c}
I d & -\mathcal{A}_{\Phi}^{-1}\left(W_{L}, W_{R}\right) \cdot S_{\widetilde{\Phi}}\left(W_{L}, W_{R}\right) \\
\hline 0 & 0
\end{array}\right] .
$$

Using this expression and dropping the $(N+1)$-th components (which are not relevant, as $\sigma$ is a known function), the numerical scheme corresponding to the decomposition (3.29) can be written as follows:

$$
\begin{aligned}
w_{i}^{n+1}= & w_{i}^{n}+\frac{\Delta t}{\Delta x}\left(F_{i-1 / 2}^{\omega}-F_{i+1 / 2}^{\omega}\right) \\
& -\frac{\Delta t}{2 \Delta x}\left(B_{i-1 / 2}\left(w_{i}^{n}-w_{i-1}^{n}\right)+B_{i+1 / 2}\left(w_{i+1}^{n}-w_{i}^{n}\right)\right) \\
& +\frac{\Delta t}{2 \Delta x}\left(S_{i-1 / 2}\left(\sigma_{i}-\sigma_{i-1}\right)+S_{i+1 / 2}\left(\sigma_{i+1}-\sigma_{i}\right)\right) \\
& +\frac{\Delta t}{2 \Delta x}\left(\omega \frac{\Delta t}{\Delta x} \mathcal{A}_{i-1 / 2}+(1-\omega) \frac{\Delta x}{\Delta t} \mathcal{A}_{i-1 / 2}^{-1}\right) S_{i-1 / 2}\left(\sigma_{i}-\sigma_{i-1}\right), \\
& -\frac{\Delta t}{2 \Delta x}\left(\omega \frac{\Delta t}{\Delta x} \mathcal{A}_{i+1 / 2}+(1-\omega) \frac{\Delta x}{\Delta t} \mathcal{A}_{i+1 / 2}^{-1}\right) S_{i+1 / 2}\left(\sigma_{i+1}-\sigma_{i}\right),
\end{aligned}
$$

where

$$
\begin{aligned}
F_{i+1 / 2}^{\omega} & =\omega F_{i+1 / 2}^{l w}+(1-\omega) F_{i+1 / 2}^{l f} \\
F_{i+1 / 2}^{l w} & =\frac{1}{2}\left[F\left(w_{i}^{n}\right)+F\left(w_{i+1}^{n}\right)-\frac{\Delta t}{\Delta x} \mathcal{A}_{i+1 / 2}^{2} \cdot\left(w_{i+1}^{n}-w_{i}^{n}\right)\right] \\
F_{i+1 / 2}^{l f} & =\frac{1}{2}\left[F\left(w_{i}^{n}\right)+F\left(w_{i+1}^{n}\right)-\frac{\Delta x}{\Delta t}\left(w_{i+1}^{n}-w_{i}^{n}\right)\right] \\
\mathcal{A}_{i+1 / 2} & =\mathcal{A}_{\Phi}\left(W_{i}^{n}, W_{i+1}^{n}\right) \\
B_{i+1 / 2} & =\mathcal{B}_{\Phi}\left(W_{i}^{n}, W_{i+1}^{n}\right) \\
S_{i+1 / 2} & =S_{\widetilde{\Phi}}\left(W_{i}^{n}, W_{i+1}^{n}\right) .
\end{aligned}
$$

The last two terms of the scheme can be interpreted as the upwinding part of the source term discretization.

If, instead, the second order Lax-Wendroff scheme is chosen, the following terms have to be added to the right-hand side of (4.12):

$$
\begin{aligned}
\omega \frac{\Delta t^{2}}{4 \Delta x^{2}} \sum_{l=1}^{N}\left(\left(w_{i}^{n}-w_{i-1}^{n}\right)^{T} \cdot C_{l, i-1 / 2}^{+} \cdot\left(w_{i}^{n}-w_{i-1}^{n}\right)\right. \\
+\left(w_{i+1}^{n}-w_{i}^{n}\right)^{T} \cdot C_{l, i+1 / 2}^{-} \cdot\left(w_{i+1}^{n}-w_{i}^{n}\right) \\
+V_{l, i-1 / 2}^{+} \cdot\left(w_{i}^{n}-w_{i-1}^{n}\right)\left(\sigma_{i}-\sigma_{i-1}\right)+V_{l, i+1 / 2}^{-} \\
\quad \cdot\left(w_{i+1}^{n}-w_{i}^{n}\right)\left(\sigma_{i+1}-\sigma_{i}\right) \\
\left.+\beta_{l, i-1 / 2}^{+}\left(\sigma_{i}-\sigma_{i-1}\right)^{2}+\beta_{l, i+1 / 2}^{-}\left(\sigma_{i+1}-\sigma_{i}\right)^{2}\right) E_{l},
\end{aligned}
$$


where $\left\{E_{1}, \ldots, E_{N}\right\}$ is the canonical basis of $\mathbb{R}^{N}$ and

$$
\begin{aligned}
C_{l, i+1 / 2}^{ \pm} & =C_{l}^{ \pm}\left(W_{i}^{n}, W_{i+1}^{n}\right), \\
V_{l, i+1 / 2}^{ \pm} & =V_{l}^{ \pm}\left(W_{i}^{n}, W_{i+1}^{n}\right), \\
\beta_{l, i+1 / 2}^{ \pm} & =\beta_{l}^{ \pm}\left(w_{i}^{n}, w_{i+1}^{n}\right) .
\end{aligned}
$$

Here, $C_{l}^{ \pm}\left(W_{L}, W_{R}\right)$ represents the matrices whose $(i, j)$ entries are, respectively,

$$
\begin{aligned}
& c_{i, j}^{l,+}\left(W_{L}, W_{R}\right)=\sum_{k=1}^{N}\left(\partial_{w_{k}} b_{l, i}\left(w_{R}\right)-\partial_{w_{i}} b_{l, k}\left(w_{R}\right)\right) a_{k, j}\left(W_{L}, W_{R}\right), \\
& c_{i, j}^{l,-}\left(W_{L}, W_{R}\right)=\sum_{k=1}^{N}\left(\partial_{w_{k}} b_{l, i}\left(w_{L}\right)-\partial_{w_{i}} b_{l, k}\left(w_{L}\right)\right) a_{k, j}\left(W_{L}, W_{R}\right),
\end{aligned}
$$

where $a_{i, j}\left(W_{L}, W_{R}\right)$ and $b_{i, j}(w)$ denote respectively the $(i, j)$ entries of $\mathcal{A}_{\Phi}\left(W_{L}, W_{R}\right)$ and $\mathcal{B}(w) ; V_{l}^{ \pm}\left(W_{L}, W_{R}\right)$ are the $1 \times N$ vectors whose $j$-th components are given by

$$
\begin{array}{r}
v_{l, j}^{+}\left(W_{L}, W_{R}\right)=-\sum_{k=1}^{N}\left(\left(\partial_{w_{k}} b_{l, j}\left(w_{R}\right)-\partial_{w_{j}} b_{l, k}\left(w_{R}\right)\right) S_{k}\left(W_{L}, W_{R}\right)\right. \\
\left.+\partial_{w_{k}} S_{l}\left(w_{R}\right) a_{k, j}\left(W_{L}, W_{R}\right)\right), \\
v_{l, j}^{-}\left(W_{L}, W_{R}\right)=-\sum_{k=1}^{N}\left(\left(\partial_{w_{k}} b_{l, j}\left(w_{L}\right)-\partial_{w_{j}} b_{l, k}\left(w_{L}\right)\right) S_{k}\left(W_{L}, W_{R}\right)\right. \\
\left.+\partial_{w_{k}} S_{l}\left(w_{L}\right) a_{k, j}\left(W_{L}, W_{R}\right)\right),
\end{array}
$$

where $S_{j}(w)$ and $S_{j}\left(W_{L}, W_{R}\right)$ denote respectively the $j$-th component of $S(w)$ and $S_{\widetilde{\Phi}}\left(W_{L}, W_{R}\right)$. Finally $\beta_{l}^{ \pm}\left(W_{L}, W_{R}\right)$ are the real numbers given by

$$
\begin{aligned}
& \beta_{l}^{+}\left(W_{L}, W_{R}\right)=\sum_{k=1}^{N} \partial_{w_{k}} S_{l}\left(w_{R}\right) S_{k}\left(W_{L}, W_{R}\right), \\
& \beta_{l}^{-}\left(W_{L}, W_{R}\right)=\sum_{k=1}^{N} \partial_{w_{k}} S_{l}\left(w_{L}\right) S_{k}\left(W_{L}, W_{R}\right) .
\end{aligned}
$$

Observe that in the expression of the numerical schemes above there are always products of the form $\mathcal{A}_{i+1 / 2}^{-1} S_{i+1 / 2}$. If one of the eigenvalues of $\mathcal{A}_{i+1 / 2}$ is zero, the corresponding product makes no sense. In this case two eigenvalues of $\widetilde{\mathcal{A}}_{\tilde{\phi}}\left(W_{i}^{n}, W_{i+1}^{n}\right)$ vanish and the problem is said to be resonant. Resonant problems exhibit an additional difficulty, as weak solutions may not be uniquely determined by their initial data, so that the limiting numerical solutions may depend both on the family of paths and the numerical scheme itself. The analysis of these difficult problems is beyond the scope of this article. According to our general purpose here, we would like to introduce some easy modifications of the schemes which avoid the difficulty related to the presence of $\mathcal{A}_{i+1 / 2}^{-1}$. The strategy followed is based on a different definition of $\widehat{I}\left(W_{L}, W_{R}\right)$ in the decomposition (3.14)-(3.15). This choice will avoid the appearance of singular matrices but, at the same time, will restrict the 
well-balanced properties of the numerical scheme. Let us suppose that the numerical scheme needs to be well-balanced only for the curves of a subset $\Gamma_{0}$ of $\Gamma$. Let us suppose that the curves $\gamma \in \Gamma_{0}$ are such that, given two states $W_{L}=\left[w_{L}, \sigma_{L}\right]^{T}$, $W_{R}=\left[w_{R}, \sigma_{R}\right]^{T}$ in $\gamma$ :

- the path $\widetilde{\Phi}\left(s ; W_{L}, W_{R}\right)$ gives a parameterization of the arc of $\gamma$ linking the states;

- the Roe matrix $A_{\Phi}\left(W_{L}, W_{R}\right)$ is regular.

Let us also assume that there exists a continuous function $\tau: \Omega \rightarrow \Omega$ such that

$$
\tau(W)=W, \quad \forall W \in \gamma \in \Gamma_{0} .
$$

We propose to modify the numerical schemes by replacing $\mathcal{A}_{i+1 / 2}^{-1}$ by $\left(\mathcal{A}_{i+1 / 2}^{*}\right)^{-1}$, with

$$
\mathcal{A}_{i+1 / 2}^{*}=\mathcal{A}_{\Phi}\left(\tau\left(W_{i}^{n}\right), \tau\left(W_{i+1}^{n}\right)\right) .
$$

With this modification, the numerical scheme is still exactly well-balanced for the curves belonging to $\Gamma_{0}$ as can be easily verified. In Section 5 , these numerical schemes will be denoted by adding MI (Modified Inverse) at the end of its name: for instance, GFORCE2MI denotes the numerical scheme of the second family with $\omega=1 /(1+\mathrm{CFL})$ using the Modified Inverse $\left(\mathcal{A}_{i+1 / 2}^{*}\right)^{-1}$.

4.2. Generalized hydrostatic reconstruction. The use of the generalized hydrostatic reconstruction presented in Subsection 3.2 .2 avoids the difficulty related to the resonant problem provided that the reconstructed states satisfy a further requirement. Let us suppose that the numerical scheme is desired to be well-balanced for the curves of a subset $\Gamma_{0}$ of $\Gamma$ for which the numerical scheme is required to be well-balanced. Let us assume that there exists a family of Lipschitz-continuous curves:

$$
\widetilde{\Gamma}=\left\{C_{W} \mid W \in \Omega\right\},
$$

satisfying the properties (P1), (P2), and (P3) described in Subsection 3.2.2, Let us denote by

$$
W_{1 / 2}^{ \pm}=\left[w_{1 / 2}^{ \pm}, \sigma_{1 / 2}^{ \pm}\right]^{T}
$$

the reconstructed states. Let us finally assume that the following property is also fulfilled:

(P4) for every pair of states $W_{0}$ and $W_{1}$, the reconstructed states are such that

$$
\sigma_{1 / 2}^{-}=\sigma_{1 / 2}^{+} .
$$

If the reconstruction operator is now applied to the family of path-conservative numerical schemes corresponding to the decomposition (3.29), the modified scheme (3.19) can be written as follows:

$$
\begin{aligned}
w_{i}^{n+1} & =w_{i}^{n}+\frac{\Delta t}{\Delta x}\left(F_{i-1 / 2}^{\omega}-F_{i+1 / 2}^{\omega}\right) \\
- & \frac{\Delta t}{2 \Delta x}\left(B_{i-1 / 2}\left(w_{i-1 / 2}^{+}-w_{i-1 / 2}^{-}\right)+B_{i+1 / 2}\left(w_{i+1 / 2}^{+}-w_{i+1 / 2}^{-}\right)\right) \\
- & \frac{\Delta t}{\Delta x}\left(\int_{0}^{1} B\left(P_{i-1 / 2}^{+}(s)\right) \frac{d P_{i-1 / 2}^{+}}{d s} d s+\int_{0}^{1} B\left(P_{i+1 / 2}^{-}(s)\right) \frac{d P_{i+1 / 2}^{-}}{d s} d s\right) \\
+ & \frac{\Delta t}{\Delta x}\left(\int_{0}^{1} S\left(P_{i-1 / 2}^{+}(s)\right) \frac{d p_{\sigma, i-1 / 2}^{+}}{d s} d s+\int_{0}^{1} S\left(P_{i+1 / 2}^{-}(s)\right) \frac{d p_{\sigma, i+1 / 2}^{-}}{d s} d s\right),
\end{aligned}
$$


where the flux $F_{i+1 / 2}^{\omega}$ and the intermediate matrices are given again by (4.12)-(4.16) but they are now evaluated at the reconstructed states $w_{i+1 / 2}^{ \pm}$. Finally,

$$
s \in[0,1] \mapsto\left[P_{i+1 / 2}^{ \pm}, p_{\sigma, i+1 / 2}^{ \pm}\right]^{T}
$$

are the chosen parameterizations of the arcs of the curves $C_{W_{i}^{n}}$ and $C_{W_{i+1}^{n}}$, linking the pairs of states $\left(W_{i}^{n}, W_{i+1 / 2}^{-}\right)$and $\left(W_{i+1 / 2}^{+}, W_{i+1}^{n}\right)$.

If, instead, the second family is used, some terms similar to (4.18) have to be added. In this case, they are also evaluated at the reconstructed states, so that all the terms related to the source term vanish.

\section{NumericAl TESTS}

In this section, the well-balanced properties, accuracy and efficiency of the numerical schemes introduced here are tested for the one-layer and the two-layer shallow water systems. In Subsections 5.1 and 5.2 we recall the formulation of these systems as well as the Roe linearizations considered here to derive the different numerical schemes. In order to avoid the appearance of negative values of the thickness of the water layers when wet/dry fronts are present, the corrections proposed in [5] have been applied.

Following [7, 20, 12, we have also derived some third order extensions of all the first order numerical schemes presented here. These extensions are based on the third order reconstruction operator PHM (piecewise hyperbolic method) introduced in [18]. For the one-layer and two-layer shallow water systems the implementation of these high order extensions can be done in such a way that the well-balanced properties of the first order schemes for the water at rest and vacuum solutions are preserved (see [12] for details). A third order TVD-Runge-Kutta discretization has been used here for the time-stepping. The high order extensions of the previous numerical schemes will be denoted by adding $\mathrm{HO}$ at the beginning of its name: for instance, HOGFORCE2MI denotes the high order extension of the numerical scheme of the second family with $\omega=1 /(1+\mathrm{CFL})$ using the modified inverse.

A large number of numerical tests has been performed to compare all of the numerical schemes introduced in this article with Roe schemes. The following general facts have been observed:

- In all cases, the Roe scheme gives the best results, followed by the GFORCE schemes. The results obtained by FORCE schemes are very close to those obtained by the GFORCE family.

- No significant differences can be found between the numerical results obtained with a numerical scheme based on the first order extension of the Lax-Wendroff scheme or on the second order one. As a consequence, it seems that it is not worth adding the extra terms (4.18).

- No significant differences can be found between the numerical results obtained by applying the Modified Inverse or the Hydrostatic Reconstruction technique.

- No significant differences can be found between the numerical results obtained with the third order extensions of the numerical schemes.

Due to this, we only show hereafter the results obtained with ROE, LAXF1MI, GFORCE1MI and their high order extensions in order to avoid an excess of tables and figures. 
5.1. Shallow water equations with depth variations. We consider the following formulation of the shallow water system:

$$
\left\{\begin{array}{l}
\frac{\partial h}{\partial t}+\frac{\partial q}{\partial x}=0 \\
\frac{\partial q}{\partial t}+\frac{\partial}{\partial x}\left[\frac{q^{2}}{h}+\frac{g}{2} h^{2}\right]=g h \frac{\partial H}{\partial x} .
\end{array}\right.
$$

The variable $x$ makes reference to the axis of the channel and $t$ is time; $q(x, t)$ and $h(x, t)$ represent the mass-flow and the thickness, respectively; $g$ is the acceleration due to gravity; $H(x)$ is the depth measured from a fixed level of reference; and $\eta=h-H$ is the free surface elevation.

This system can be written as a particular case of (4.1) with $N=2, \mathcal{B}=0$, $\sigma=H$ and

$$
w=\left(\begin{array}{c}
h \\
q
\end{array}\right), \quad F(w)=\left(\begin{array}{c}
q \\
\frac{q^{2}}{h}+\frac{g}{2} h^{2}
\end{array}\right), \quad S(w)=\left(\begin{array}{c}
0 \\
g h
\end{array}\right) .
$$

The eigenvalues of this system are:

$$
\lambda_{1}=u-c, \lambda_{2}=u+c,
$$

where $u=q / h$ represents the averaged velocity and $c=\sqrt{g h}$. A state $W$ is said to be supercritical, critical or subcritical if $F_{r}>1, F_{r}=1$, or $F_{r}<1$, respectively,

being the Froude number.

$$
F_{r}=\frac{u}{c}
$$

The integral curves of the linearly degenerate field are given by the equations:

$$
q=\text { constant }, \quad h+\frac{q^{2}}{2 g h^{2}}-H=\text { constant } .
$$

In the particular case $q=0$ these curves are straight lines in $h, q, H$ space:

$$
q=0, \quad h-H=\text { constant } .
$$

These curves correspond to water at rest solutions, while vacuum solutions correspond to:

$$
h=0, \quad q=0 .
$$

We consider here the following Roe matrices based on the family of segments (see [21]):

$$
\widetilde{\mathcal{A}}_{i+1 / 2}=\left[\begin{array}{c|c}
A_{i+1 / 2} & S_{i+1 / 2} \\
\hline 0 \ldots 0 & 0
\end{array}\right]
$$

where

$$
A_{i+1 / 2}=\left[\begin{array}{cc}
1 & 0 \\
-\left(u_{i+1 / 2}^{n}\right)^{2}+\left(c_{i+1 / 2}^{n}\right)^{2} & 2 u_{i+1 / 2}^{n}
\end{array}\right], \quad S_{i+1 / 2}=\left[\begin{array}{c}
0 \\
\left(c_{i+1 / 2}^{n}\right)^{2}
\end{array}\right],
$$

with

$$
u_{i+1 / 2}^{n}=\frac{\sqrt{h_{i}^{n}} u_{i}^{n}+\sqrt{h_{i+1}^{n}} u_{i+1}^{n}}{\sqrt{h_{i}^{n}}+\sqrt{h_{i+1}^{n}}}, \quad c_{i+1 / 2}^{n}=\sqrt{g \frac{h_{i}^{n}+h_{i+1}^{n}}{2}} .
$$

The corresponding Roe scheme coincides with the generalized $Q$-scheme of Roe upwinding the source term introduced in 2. It can be easily verified that the 
hypotheses of Proposition 2.2 are satisfied for any curve $\gamma$ given by (5.3). Therefore, the Roe scheme is exactly well-balanced for water at rest or vacuum stationary solutions. Moreover, it is well-balanced of order 2 for general stationary solutions (see [21]).

To apply the technique of modification of the inverse, we consider the subset $\Gamma_{0}$ of the integral curves of the linearly degenerate field defined by (5.3) together with the projection:

$$
W=\left[\begin{array}{c}
h \\
q \\
H
\end{array}\right] \rightarrow \tau(W)=\left[\begin{array}{c}
h \\
0 \\
H
\end{array}\right]
$$

With this choice, it can be easily shown that

$$
\mathcal{A}_{i+1 / 2}^{*}=\left[\begin{array}{cc}
0 & 1 \\
\left(c_{i+1 / 2}^{n}\right)^{2} & 0
\end{array}\right] .
$$

This matrix is regular if $c_{i+1 / 2}^{n}>0$. The corresponding numerical schemes are wellbalanced for water at rest solutions. Nevertheless, the second order of accuracy for general stationary solutions of Roe methods is not preserved.

The hydrostatic reconstruction technique has been used in 1 to obtain first and second order schemes for the shallow water system which are well-balanced for vacuum and water at rest solutions. It has also been used in 9] to obtain high order Roe schemes which are also well-balanced for the vacuum and water at rest solutions. A first order Roe method which is well-balanced for every smooth stationary solution has also been obtained in the latter reference. A different generalization of the hydrostatic reconstruction technique has also been used in [22] to obtain high order numerical schemes which are well-balanced for every smooth stationary solution.

5.2. The two-layer shallow water system. We consider here the following formulation of the two-layer shallow water system (see [8]):

$$
\left\{\begin{array}{l}
\frac{\partial h_{1}}{\partial t}+\frac{\partial q_{1}}{\partial x}=0 \\
\frac{\partial q_{1}}{\partial t}+\frac{\partial}{\partial x}\left(\frac{q_{1}^{2}}{h_{1}}+\frac{g}{2} h_{1}^{2}\right)=-g h_{1} \frac{\partial h_{2}}{\partial x}+g h_{1} \frac{d H}{d x} \\
\frac{\partial h_{2}}{\partial t}+\frac{\partial q_{2}}{\partial x}=0 \\
\frac{\partial q_{2}}{\partial t}+\frac{\partial}{\partial x}\left(\frac{q_{2}^{2}}{h_{2}}+\frac{g}{2} h_{2}^{2}\right)=-\frac{\rho_{1}}{\rho_{2}} g h_{2} \frac{\partial h_{1}}{\partial x}+g h_{2} \frac{d H}{d x}
\end{array} .\right.
$$

In these equations, index 1 makes reference to the upper layer and index 2 to the lower one layer. The fluid is assumed to occupy a straight channel with constant rectangular cross section and constant width. The coordinate $x$ refers to the axis of the channel, $t$ is time, and $g$ is the acceleration due to gravity. $H(x)$ represents the depth function measured from a fixed level of reference. Each layer is assumed to have a constant density, $\rho_{i}, i=1,2\left(\rho_{1}<\rho_{2}\right)$. The unknowns $q_{i}(x, t)$ and $h_{i}(x, t)$ represent respectively the mass-flow and the thickness of the $i$-th layer at the section of coordinate $x$ at time $t$. 
System (5.7) can be written in the form (4.1) with $N=4, \sigma=H$,

$$
\begin{gathered}
w(x, t)=\left[\begin{array}{c}
h_{1}(x, t) \\
q_{1}(x, t) \\
h_{2}(x, t) \\
q_{2}(x, t)
\end{array}\right], \quad F(w)=\left[\begin{array}{c}
q_{1} \\
\frac{q_{1}^{2}}{h_{1}}+\frac{g}{2} h_{1}^{2} \\
q_{2} \\
\frac{q_{2}^{2}}{h_{2}}+\frac{g}{2} h_{2}^{2}
\end{array}\right], \quad S(w)=\left[\begin{array}{c}
0 \\
g h_{1} \\
0 \\
g h_{2}
\end{array}\right], \\
\mathcal{B}(w)=\left[\begin{array}{cccc}
0 & 0 & 0 & 0 \\
0 & 0 & g h_{1} & 0 \\
0 & 0 & 0 & 0 \\
g r h_{2} & 0 & 0 & 0
\end{array}\right],
\end{gathered}
$$

where

$$
r=\frac{\rho_{1}}{\rho_{2}} .
$$

The characteristic equation of the homogeneous system, i.e. $H=$ constant, is:

$$
\left(\lambda^{2}-2 u_{1} \lambda+u_{1}^{2}-g h_{1}\right)\left(\lambda^{2}-2 u_{2} \lambda+u_{2}^{2}-g h_{2}\right)=r g^{2} h_{1} h_{2}
$$

where $u_{i}=q_{i} / h_{i}$ represents the averaged velocity of the $i$-th layer and $c_{i}=\sqrt{g h_{i}}$, $i=1,2$.

In the case $r \cong 1$ (which is the situation arising in many geophysical flows) a first-order approximation of the eigenvalues was given in 24]:

$$
\begin{aligned}
& \lambda_{\text {ext }}^{ \pm} \cong \frac{u_{1} h_{1}+u_{2} h_{2}}{h_{1}+h_{2}} \pm\left(g\left(h_{1}+h_{2}\right)\right)^{\frac{1}{2}} \\
& \lambda_{\text {int }}^{ \pm} \cong \frac{u_{1} h_{2}+u_{2} h_{1}}{h_{1}+h_{2}} \pm\left(g^{\prime} \frac{h_{1} h_{2}}{\left(h_{1}+h_{2}\right)}\left[1-\frac{\left(u_{1}-u_{2}\right)^{2}}{g^{\prime}\left(h_{1}+h_{2}\right)}\right]\right)^{\frac{1}{2}} .
\end{aligned}
$$

In the above expression, $g^{\prime}$ is the reduced gravity:

$$
g^{\prime}=(1-r) g
$$

Note that, $\lambda_{\text {int }}^{ \pm}$may become complex, corresponding to the development of shear instabilities. We note that an approximated hyperbolicity condition that is regarded as acceptable when the ratio of densities $r$ is close to 1 , as happens in the oceanographical applications, is

$$
\frac{\left(u_{1}-u_{2}\right)^{2}}{g(1-r)\left(h_{1}+h_{2}\right)}<1 .
$$

In the Strait of Gibraltar, $r$ is about 0.99805 .

In the present work only the case where $\lambda_{\text {int }}^{ \pm} \in \mathbb{R}$ is considered; i.e., the flow is supposed to be stable and the system strictly hyperbolic.

The integral curves of the 5-th characteristic field are:

$$
\begin{gathered}
q_{1}=\text { constant }, \quad \frac{u_{1}^{2}}{2}-\frac{u_{2}^{2}}{2}+g_{r} h_{1}=\text { constant }, \\
q_{2}=\text { constant }, \quad \frac{u_{1}^{2}}{2}+g\left(h_{1}+h_{2}-H\right)=\text { constant } .
\end{gathered}
$$

In particular, if $q_{1}=0, q_{2}=0$, we obtain the solutions:

$$
q_{1}=0, h_{1}(x)=\text { constant }, q_{2}=0, h_{2}(x)-H(x)=\text { constant },
$$


representing water at rest. Another steady state solution corresponding to a vacuum is given by

$$
h_{i}=0, \quad q_{i}=0, \quad i=1,2 .
$$

We consider again the family of segments in order to construct a Roe linearization (see [21] for details):

$$
\widetilde{\mathcal{A}}_{i+1 / 2}=\left[\begin{array}{c|c}
A_{i+1 / 2} & S_{i+1 / 2} \\
\hline 0 \ldots 0 & 0
\end{array}\right]
$$

where $A_{i+1 / 2}=$

$$
\begin{gathered}
{\left[\begin{array}{cccc}
0 & 1 & 0 & 0 \\
-\left(u_{1, i+1 / 2}^{n}\right)^{2}+\left(c_{1, i+1 / 2}^{n}\right)^{2} & 2 u_{1, i+1 / 2}^{n} & \left(c_{1, i+1 / 2}^{n}\right)^{2} & 0 \\
0 & 0 & 0 & 1 \\
r\left(c_{2, i+1 / 2}^{n}\right)^{2} & 0 & -\left(u_{2, i+1 / 2}^{n}\right)^{2}+\left(c_{2, i+1 / 2}^{n}\right)^{2} & 2 u_{2, i+1 / 2}^{n}
\end{array}\right]} \\
S_{i+1 / 2}=\left[\begin{array}{c}
0 \\
-\left(c_{1, i+1 / 2}^{n}\right)^{2} \\
0 \\
-\left(c_{2, i+1 / 2}^{n}\right)^{2}
\end{array}\right]
\end{gathered}
$$

Here,

$$
u_{k, i+1 / 2}^{n}=\frac{\sqrt{h_{k, i}^{n}} u_{k, i}^{n}+\sqrt{h_{k, i+1}^{n}} u_{k, i+1}^{n}}{\sqrt{h_{k, i}^{n}}+\sqrt{h_{k, i+1}^{n}}}, \quad c_{k, i+1 / 2}^{n}=\sqrt{g \frac{h_{k, i}^{n}+h_{k, i+1}^{n}}{2}}, k=1,2 .
$$

The corresponding Roe scheme coincides with the generalized $Q$-scheme of Roe upwinding the source term introduced in [8. It is well-balanced of order 2 for general stationary solutions and exactly well-balanced for water at rest or vacuum stationary solutions.

In order to overcome the difficulty of the calculation of the inverse matrix in resonant cases, we consider the set of integral curves of the linearly degenerate field (5.11) together with the projection:

$$
W=\left[\begin{array}{c}
h_{1} \\
q_{1} \\
h_{2} \\
q_{2} \\
H
\end{array}\right] \rightarrow \tau(W)=\left[\begin{array}{c}
h_{1} \\
0 \\
h_{2} \\
0 \\
H
\end{array}\right] .
$$

With this choice, we have

$$
\mathcal{A}_{i+1 / 2}^{*}=\left[\begin{array}{cccc}
0 & 1 & 0 & 0 \\
\left(c_{1, i+1 / 2}^{n}\right)^{2} & 0 & \left(c_{1, i+1 / 2}^{n}\right)^{2} & 0 \\
0 & 0 & 0 & 1 \\
r\left(c_{2, i+1 / 2}^{n}\right)^{2} & 0 & \left(c_{2, i+1 / 2}^{n}\right)^{2} & 0
\end{array}\right],
$$

which is regular.

The hydrostatic reconstruction technique can also be extended in this case to obtain numerical schemes which are well-balanced for water at rest or vacuum stationary state solutions (see 9] for details). 
TABle 1. Test 1: Water at rest solution. Errors.

\begin{tabular}{||c||c|c||c|c||c|c||}
\hline \multicolumn{2}{c|}{} & \multicolumn{2}{c|}{ ROE } & \multicolumn{2}{c||}{ LAXF1MI } & \multicolumn{2}{c||}{ GFORCE1MI } \\
\cline { 2 - 7 } & $L_{1} h$ & $L_{1} q$ & $L_{1} h$ & $L_{1} q$ & $L_{1} h$ & $L_{1} q$ \\
\hline Cells & error & error & error & error & error & error \\
\hline \hline 100 & $1.12 E^{-15}$ & $1.75 E^{-15}$ & $2.23 E^{-15}$ & $2.17 E^{-15}$ & $1.78 E^{-15}$ & $2.05 E^{-15}$ \\
\hline
\end{tabular}
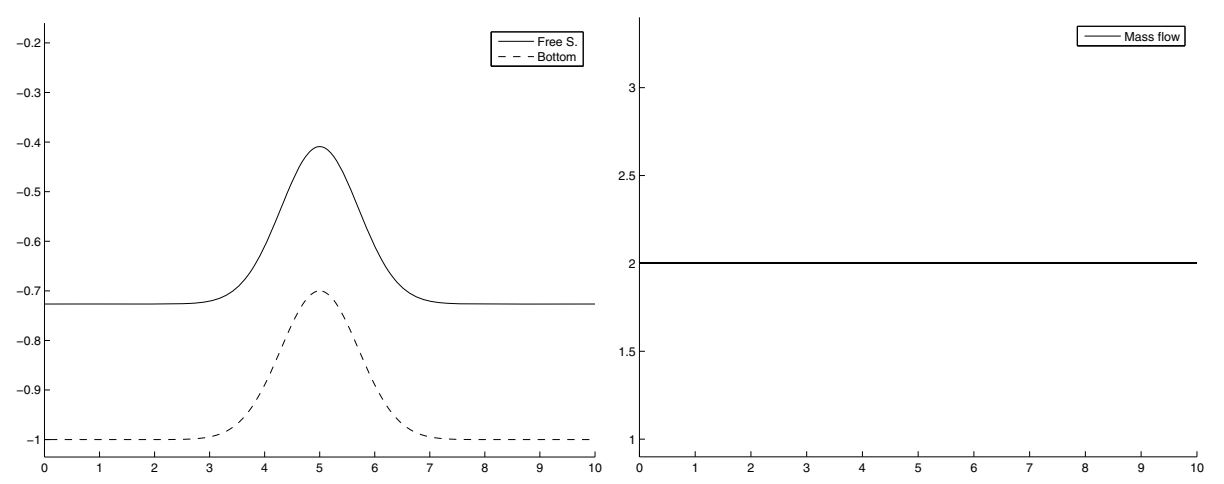

Figure 1. Test 1: Stationary solution and depth function.

TABle 2. Test 1: Errors and order.

\begin{tabular}{|c|c|c|c|c|c|c|c|c|}
\hline \multirow[b]{3}{*}{ Cells } & \multicolumn{4}{|c|}{ ROE } & \multicolumn{4}{|c|}{ LAXF1MI } \\
\hline & \multicolumn{2}{|c|}{$L_{1} h$} & \multicolumn{2}{|c|}{$L_{1} q$} & \multicolumn{2}{|c|}{$L_{1} h$} & \multicolumn{2}{|c|}{$L_{1} q$} \\
\hline & error & order & error & order & error & order & error & order \\
\hline$\overline{10}$ & $2.10 E^{-03}$ & - & $1.55 E^{-15}$ & - & $9.97 E^{-01}$ & - & $1.97 E^{+01}$ & - \\
\hline 20 & $6.23 E^{-04}$ & 1.75 & $1.60 E^{-15}$ & - & 1.17 & -0.23 & $1.93 E^{+01}$ & 0.03 \\
\hline 40 & $1.58 E^{-04}$ & 1.97 & $1.27 E^{-15}$ & - & $2.64 E^{-01}$ & 2.15 & $8.09 E^{-01}$ & 4.58 \\
\hline 80 & $3.98 E^{-05}$ & 1.99 & $1.30 E^{-15}$ & - & $1.26 E^{-01}$ & 1.06 & $3.97 E^{-01}$ & 1.03 \\
\hline \multirow[t]{3}{*}{160} & $9.95 E^{-06}$ & 2.00 & $1.38 E^{-15}$ & - & $6.19 E^{-02}$ & 1.03 & $1.97 E^{-01}$ & 1.01 \\
\hline & \multicolumn{4}{|c|}{ GFORCE1MI } & & & & \\
\hline & \multicolumn{2}{|c|}{$L_{1} h$} & \multicolumn{2}{|c|}{$L_{1} q$} & & & & \\
\hline Cells & error & order & error & order & & & & \\
\hline 10 & 9.50 & $\begin{array}{c}- \\
-\end{array}$ & $2.12 E^{+01}$ & - & & & & \\
\hline 20 & $2.47 E^{-01}$ & 5.27 & $6.94 E^{-01}$ & 4.93 & & & & \\
\hline 40 & $1.18 E^{-01}$ & 1.07 & $3.75 E^{-01}$ & 0.89 & & & & \\
\hline 80 & $5.85 E^{-02}$ & 1.01 & $1.86 E^{-01}$ & 1.01 & & & & \\
\hline 160 & $2.92 E^{-02}$ & 1.00 & $9.34 E^{-02}$ & 0.99 & & & & \\
\hline
\end{tabular}

5.3. Test 1: One-layer system. Well-balanced property. All the numerical schemes considered in this work solve exactly water at rest solutions. The objective of this first test case is to check this fact numerically. To do it, a channel whose axis is the interval $[0,1]$ and whose depth function $H(x)$ is given by an exponential perturbed with a random noise is considered. As initial conditions we take $h(x, 0)=$ 


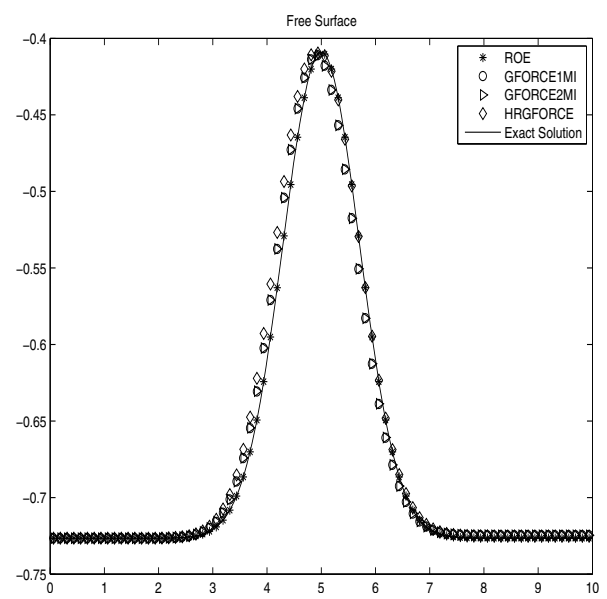

(a) Free surface

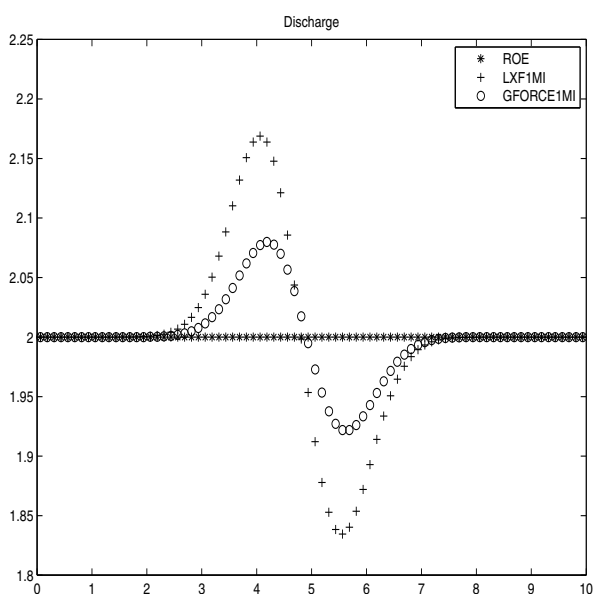

(b) Discharge

Figure 2. Test 1: Comparison with the exact solution at $t=100$ with $\Delta x=1 / 8$.

$H(x)$ and $q(x, 0)=0, x \in[0,1]$. Table 1 shows the $L^{1}$ errors obtained with the numerical schemes: ROE, LAXF1MI and GFORCE1MI for a given mesh with $\Delta x=0.01$. The CFL parameter is set to 0.9. As expected, all numerical schemes preserve the steady state up to machine accuracy. Similar results are obtained by the high order extensions of the previous numerical schemes.

Next, we test the well-balanced property for smooth stationary solutions with $q \neq 0$. We consider a channel whose axis is the interval $[0,10]$ and whose bathymetry is given by the function $H(x)=1-0.3 e^{-(x-5)^{2}}$. We consider the supercritical solution corresponding to the integral curve

$$
q=2, h-H+\frac{q^{2}}{2 g h^{2}}=2 .
$$

(See Figure 11).

Table 2 shows the $L^{1}$ errors obtained with the numerical schemes: ROE, LAXF1MI and GFORCE1MI for five regular meshes with increasing number of cells. The CFL parameter is set to 0.9. As expected, ROE gives order 2 while the other numerical schemes only achieve first order. Figures 2(a) and 2(b) show the comparison of the numerical results with the exact solution at time $t=100$ with $\Delta x=1 / 8$. As expected, the best results are given by ROE, and GFORCE1MI provides better results than LAXF1MI.

5.4. Test 2: One-layer system. Well-balanced property for a nonsmooth solution. This test is designed to assess the long time behavior and the convergence to a steady state including a regular transition and a shock. The axis of the channel is the interval $[0,25]$. The bottom topography is given by the function

$$
H(x)= \begin{cases}0.05(x-10)^{2}, & \text { if } 8<x<12 \\ 0.2, & \text { otherwise }\end{cases}
$$




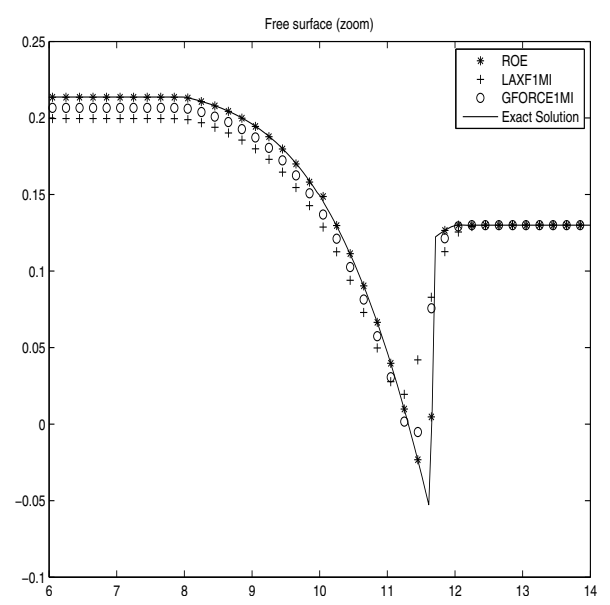

(a) Free surface

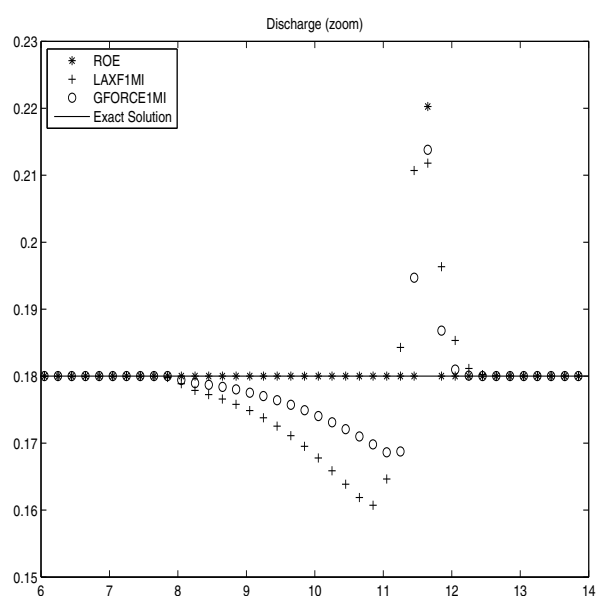

(b) Discharge

Figure 3. Test 2: Comparison with the reference solution at $t=$ 200 with $\Delta x=1 / 8$.

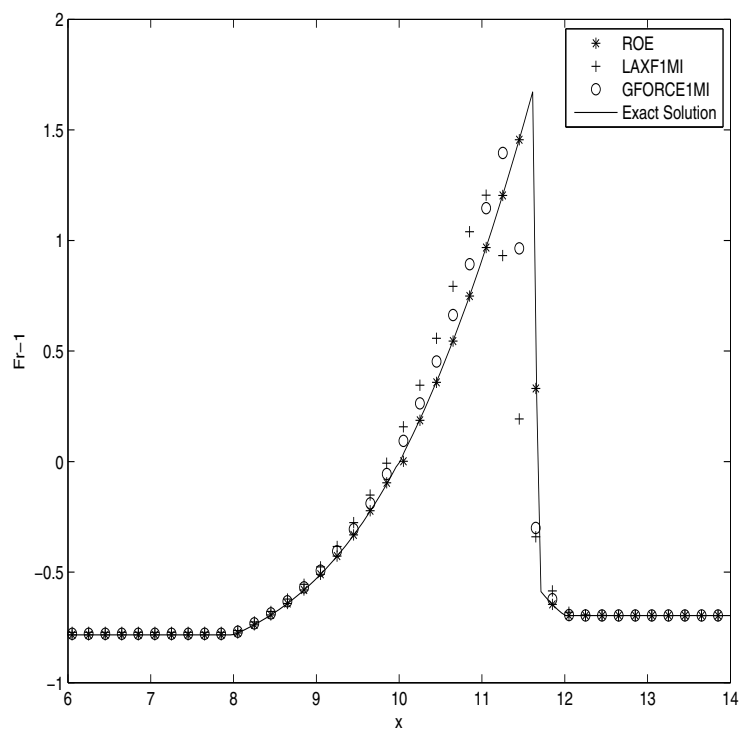

Figure 4. Test 2: $F_{r}-1$. Comparison with the reference solution at $t=200$ with $\Delta x=1 / 8$.

The initial conditions are $h(x, 0)=0.13+H(x), q(x, 0)=0.18$, and the boundary conditions are $q(0, t)=0.18, h(25, t)=0.33$. The CFL parameter is set to 0.9 .

A reference solution is computed with a mesh of 3200 points. Table 3 shows the $L^{1}$ errors obtained with ROE, LAXF1MI, and GFORCE1MI for five regular meshes with increasing number of cells. In this case, as the steady state solution 
TABLE 3. Test 2: Errors and order at $t=200$.

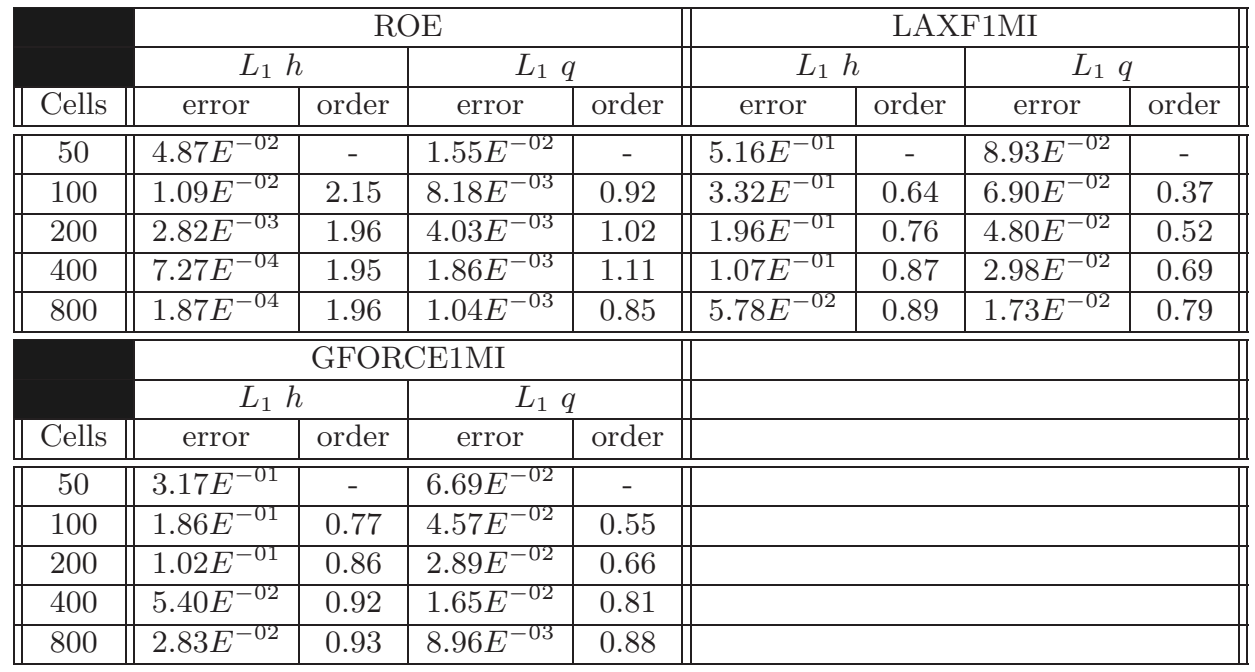

is not regular, at most first order approximation could be expected for all the numerical schemes. As happened in the previous test case, ROE is the most accurate scheme. Figures 3(a) and 3(b) show the comparison of the numerical results with the reference solution at time $t=200$ with $\Delta x=1 / 8$. The conclusions coincide with those of the previous test case.

Figure 4 shows $F_{r}-1, F_{r}$ being the Froude number, computed from the numerical solution obtained using ROE, LAXF1MI, and GFORCE1MI schemes and the reference solution. As can be observed, this numerical test corresponds to a nonsmooth transcritical solution. Note that if the numerical scheme proposed in 22 was used, as it is well-balanced for any stationary solution, then the corresponding errors in Tables 2 and 3 would be of the order of machine accuracy.

5.5. Test 3: Two-layer shallow water. Well-balanced property. In this numerical experiment we test the well-balanced property of the numerical schemes for smooth steady solutions not corresponding to water at rest, that is, $q_{1} \neq 0$ and $q_{2} \neq 0$. The axis of the channel is the interval $[0,10]$, and the bottom is given by the function $H(x)=2.0-0.5 e^{-(x-5)^{2}}$. The ratio of densities is set to $r=0.98$. We consider a subcritical steady solution corresponding to the integral curve (see Figure (5):

$$
q_{1}=0.15, \frac{u_{1}^{2}}{2}-\frac{u_{2}^{2}}{2}+g(1-r) h_{1}=K_{1}, q_{2}=-0.15, \frac{u_{1}^{2}}{2}+g\left(h_{1}+h_{2}-H\right)=K_{2},
$$

with

$$
\begin{gathered}
K_{1}=\frac{1}{2}\left(\frac{0.15}{0.5}\right)^{2}-\frac{1}{2}\left(\frac{0.15}{1.5}\right)^{2}+g \cdot(1-0.98) \cdot 0.5, \\
K_{2}=\frac{1}{2}\left(\frac{0.15}{0.5}\right)^{2}, g=9.81 .
\end{gathered}
$$




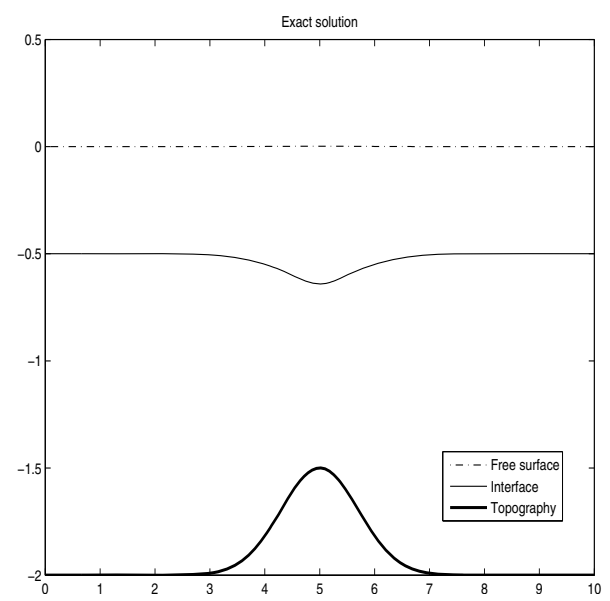

(a) Free surface and interface.

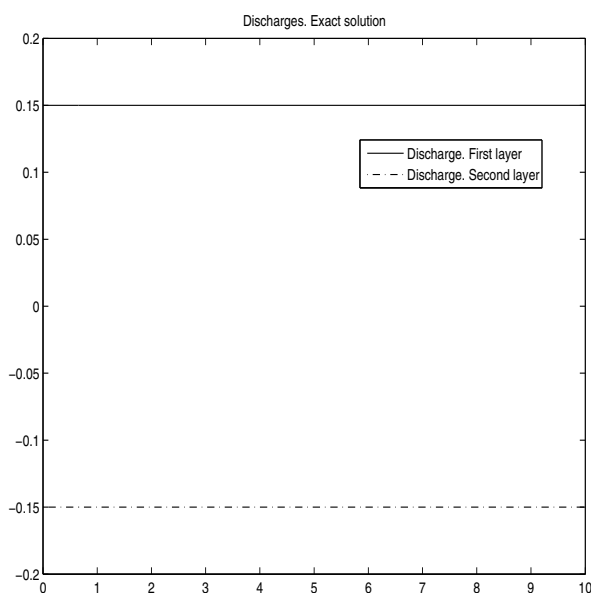

(b) Discharges.

Figure 5. Test 3: Exact solution

TABle 4. Test 3: Errors and order

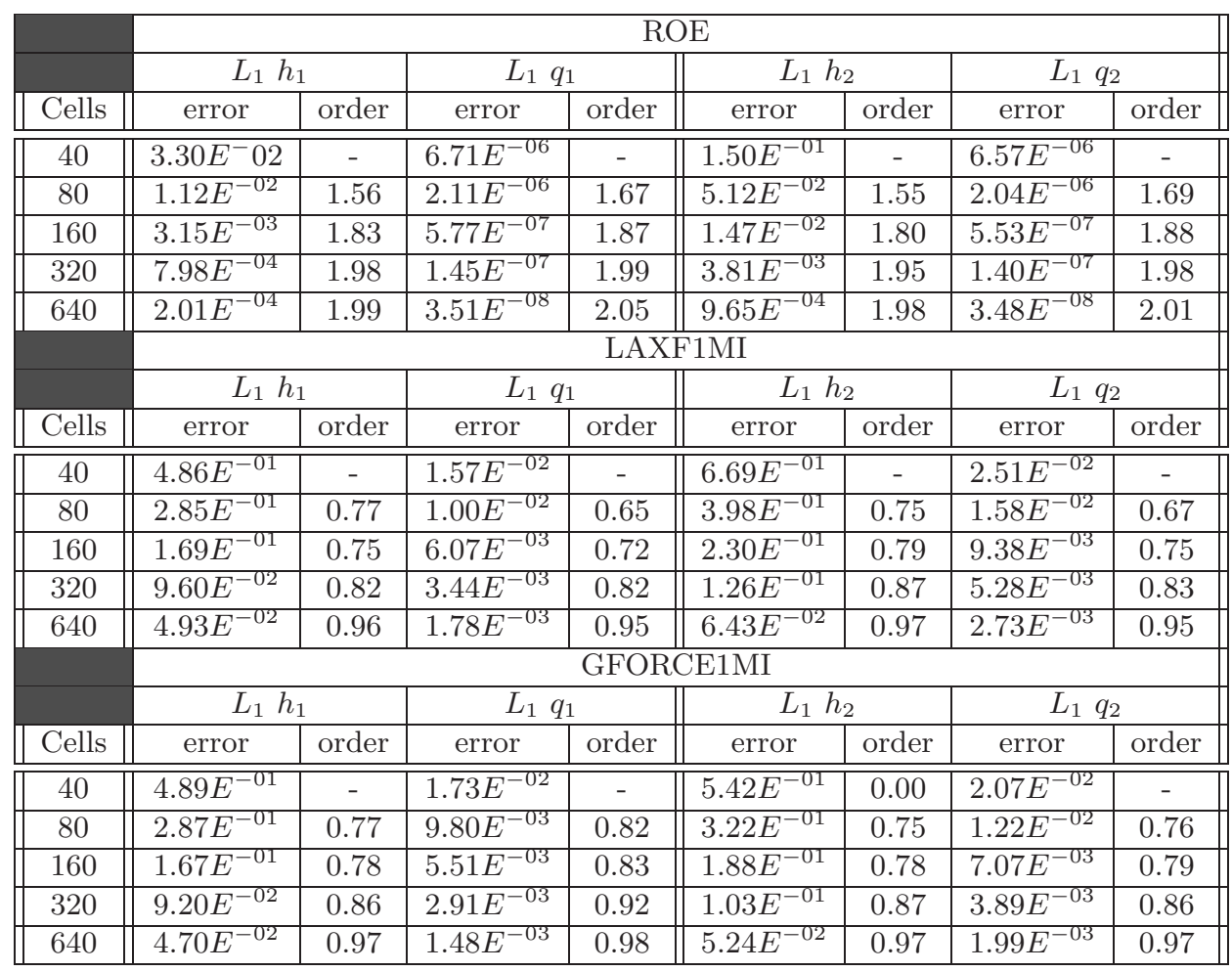




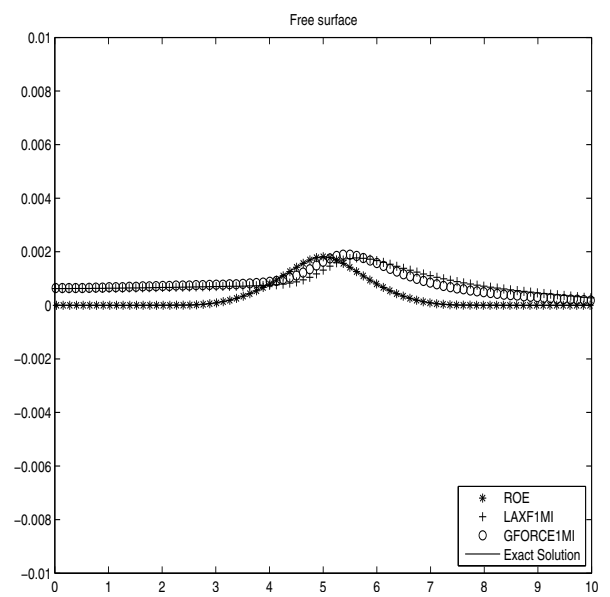

(a) Free surface

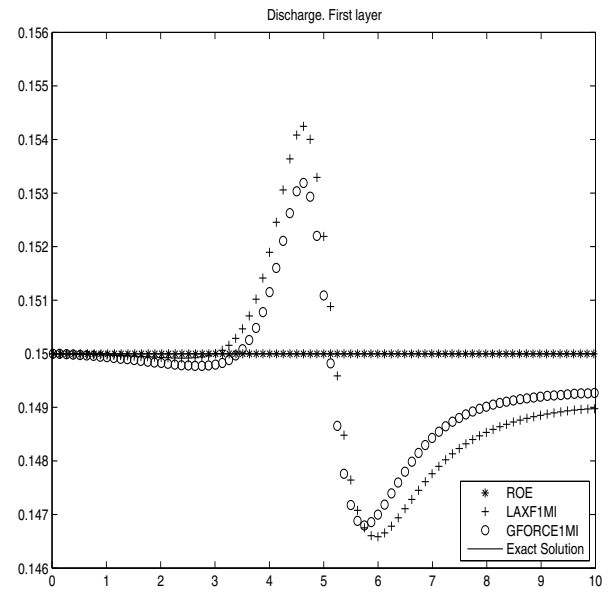

(c) Discharge. First layer

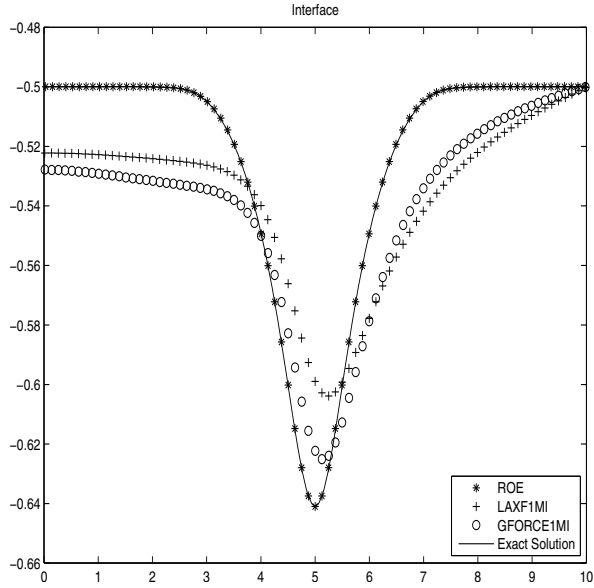

(b) Interface

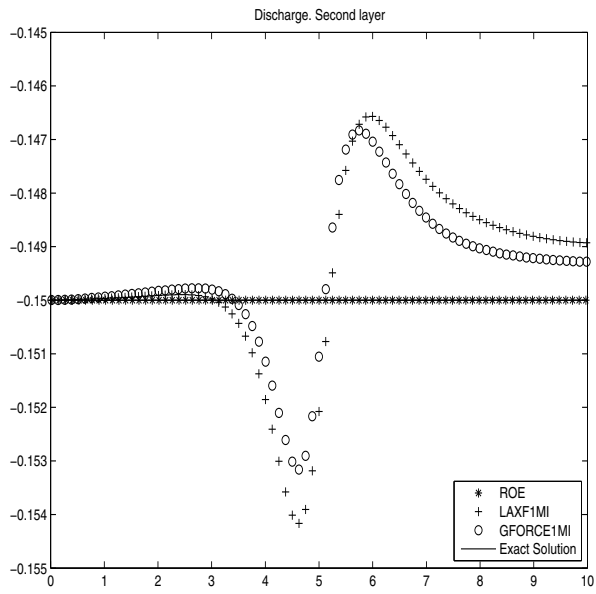

(d) Discharge. Second layer

Figure 6. Test 3: ROE, LAXF1MI and GFORCE1MI schemes. Comparison with the exact solution with $\Delta x=1 / 16$.

As boundary conditions, the discharges are both imposed at $x=0$, that is, $q_{1}(0, t)=$ 0.15 and $q_{2}(0, t)=-0.15$, while the free surface level is fixed to $z=0$ at $x=10$. The CFL parameter is set to 0.9 .

Tables 4 and 5 show the $L^{1}$ errors obtained with ROE, LAXF1MI and GFORCE1MI, and their high order extensions HOROE, HOLAXF1MI and HOGFORCE1MI, for five regular meshes with increasing number of cells. As expected, the high order numerical schemes give order 3 , ROE gives order 2 , and the other schemes only give first order accuracy.

The numerical results obtained with $\Delta x=1 / 16$ are compared with the exact solution in Figures 6 and 7 . The first one corresponds to ROE, LAXF1MI, and GFORCE1MI and the second one to HOROE, HOLAXF1MI and HOGFORCE1MI. 
TABLE 5. Test 3 (II): Errors and order

\begin{tabular}{|c|c|c|c|c|c|c|c|c|}
\hline \multirow[b]{3}{*}{ Cells } & \multicolumn{8}{|c|}{ HOROE } \\
\hline & \multicolumn{2}{|c|}{$L_{1} h_{1}$} & \multicolumn{2}{|c|}{$\begin{array}{ll}L_{1} & q_{1}\end{array}$} & \multicolumn{2}{|c|}{$L_{1} h_{2}$} & \multicolumn{2}{|c|}{$L_{1} q_{2}$} \\
\hline & error & order & error & order & error & order & error & order \\
\hline 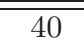 & 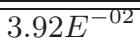 & - & $\bar{c} 5.46 E^{-06}$ & - & $1.54 E^{-01}$ & - & $7 \overline{7.99 E^{-06}}$ & - \\
\hline 80 & $4.51 E^{-03}$ & 3.12 & $6.15 E^{-07}$ & 3.15 & $1.77 E^{-02}$ & 3.12 & $8.82 E^{-07}$ & 3.18 \\
\hline 160 & $19 E^{-04}$ & 2.46 & $1.09 E^{-07}$ & 2.50 & $3.09 E^{-03}$ & 2.52 & $1.45 E^{-07}$ & 2.60 \\
\hline 320 & $26 E^{-04}$ & 2.70 & $1.54 E^{-08}$ & 2.82 & $4.59 E^{-04}$ & 2.75 & $2.02 E^{-08}$ & 2.85 \\
\hline \multirow[t]{3}{*}{640} & $1.67 E^{-05}$ & 2.92 & $1.94 E^{-09}$ & 2.99 & $6.15 E^{-05}$ & 2.90 & $2.52 E^{-09}$ & 3.00 \\
\hline & \multicolumn{8}{|c|}{ HOLAXF1MI } \\
\hline & \multicolumn{2}{|c|}{$L_{1} h_{1}$} & \multicolumn{2}{|c|}{$\begin{array}{ll}L_{1} q_{1} \\
\end{array}$} & \multicolumn{2}{|c|}{$L_{1} \quad h_{2}$} & \multicolumn{2}{|c|}{$\begin{array}{ll}L_{1} & q_{2} \\
\end{array}$} \\
\hline Cells & error & order & error & order & error & order & error & order \\
\hline$\overline{\overline{440}}$ & 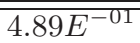 & - & $\overline{c 1.61 E^{-02}}$ & - & $6.68 E^{-01}$ & - & $2.53 E^{-02}$ & - \\
\hline 80 & $6.28 E^{-02}$ & 2.96 & $2.00 E^{-03}$ & 3.01 & $8.18 E^{-02}$ & 3.03 & $3.18 E^{-03}$ & 2.99 \\
\hline 160 & $1.10 E^{-02}$ & 2.52 & $3.46 E^{-04}$ & 2.53 & $1.41 E^{-02}$ & 2.54 & $5.18 E^{-04}$ & 2.62 \\
\hline 320 & $1.61 E^{-03}$ & 2.77 & $4.80 E^{-05}$ & 2.85 & $2.08 E^{-03}$ & 2.76 & $7.14 E^{-05}$ & 2.86 \\
\hline \multirow[t]{3}{*}{640} & $2.05 E^{-04}$ & 2.97 & $6.04 E^{-06}$ & 2.99 & $2.63 E^{-04}$ & 2.98 & $8.98 E^{-06}$ & 2.99 \\
\hline & \multicolumn{8}{|c|}{ HOGFORCE1MI } \\
\hline & \multicolumn{2}{|c|}{$L_{1} h_{1}$} & \multicolumn{2}{|c|}{$L_{1} q_{1}$} & \multicolumn{2}{|c|}{$L_{1} h_{2}$} & \multicolumn{2}{|c|}{$L_{1} q_{2}$} \\
\hline Cells & error & order & error & order & error & order & error & order \\
\hline$\overline{40}$ & $4.87 E^{-01}$ & - & $1.76 E^{-02}$ & - & $5.39 E^{-01}$ & - & $2.10 E^{-02}$ & - \\
\hline 80 & $6.17 E^{-02}$ & 2.98 & $2.26 E^{-03}$ & 2.96 & $6.69 E^{-02}$ & 3.01 & $2.63 E^{-03}$ & 3.00 \\
\hline 160 & $1.08 E^{-02}$ & 2.52 & $3.86 E^{-04}$ & 2.55 & $1.14 E^{-02}$ & 2.55 & $4.33 E^{-04}$ & 2.60 \\
\hline 320 & $1.62 E^{-03}$ & 2.73 & $5.47 E^{-05}$ & 2.82 & $1.69 E^{-03}$ & 2.76 & $6.01 E^{-05}$ & 2.85 \\
\hline 640 & $2.11 E^{-04}$ & 2.94 & $6.88 E^{-06}$ & 2.99 & $2.17 E^{-04}$ & 2.96 & $7.61 E^{-06}$ & 2.98 \\
\hline
\end{tabular}

TABle 6. Test 3: CPU time (in seconds).

\begin{tabular}{||c||c|c|c|c|c|c||}
\hline Cells & ROE & LAXF1MI & GFORCE1MI & HOROE & HOLAXF1MI & HOGFORCE1MI \\
\hline \hline 40 & 4.27 & 0.94 & 1.35 & 23.90 & 10.64 & 14.47 \\
80 & 16.56 & 3.68 & 5.22 & 95.60 & 43.11 & 58.54 \\
160 & 65.48 & 14.49 & 20.85 & 394.90 & 184.07 & 233.93 \\
320 & 258.60 & 57.30 & 81.83 & 1567.40 & 754.96 & 946.99 \\
\hline
\end{tabular}

Finally, Table [ shows the CPU time (in seconds) used to compute the solution at $t=300$ with ROE, LAXF1MI and GFORCE1MI schemes and their high order extensions. It can be observed that the CPU time is reduced up to 4.5 times when using the LAXF1MI or 3.15 times when using GFORCE1MI in comparison with $\mathrm{ROE}$, which gives the best results. The CPU time is reduced up to 2.10 times when using HOLAXF1MI or 1.65 times when using HOGFORCE1MI in comparison with HOROE. These reductions are due to the fact that, while in the implementation of ROE, the eigenvalues of the intermediate matrices are numerically computed, for LAXF1MI and GFORCE1MI only an estimate of the maximum eigenvalue is required to fit the CFL condition. The same thing happens for the high order 


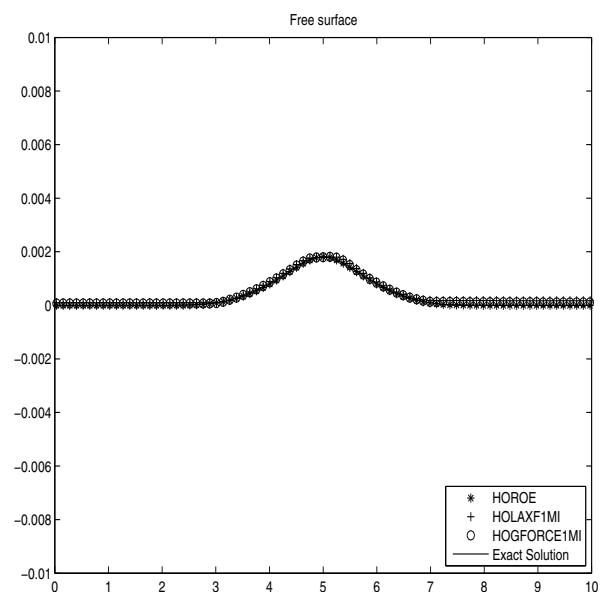

(a) Free surface

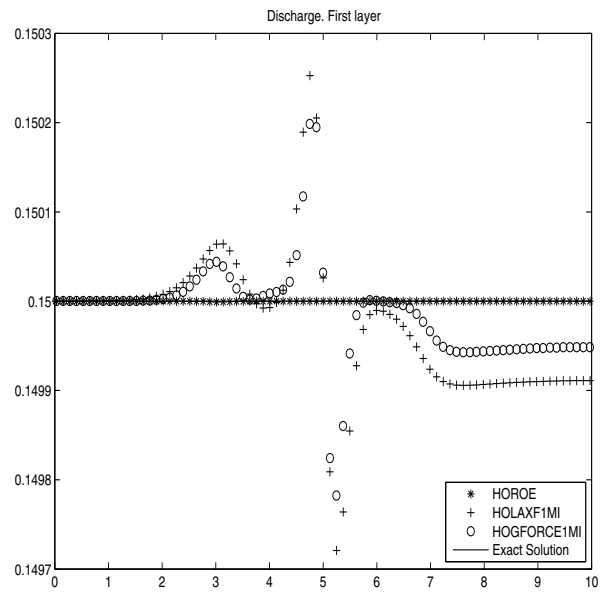

(c) Discharge. First layer

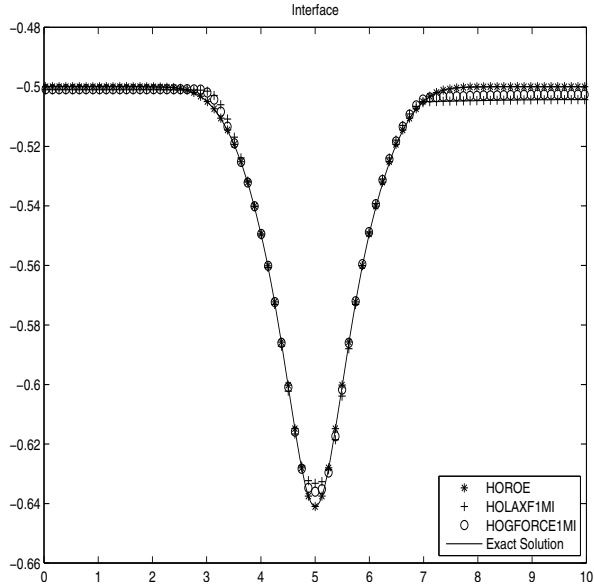

(b) Interface

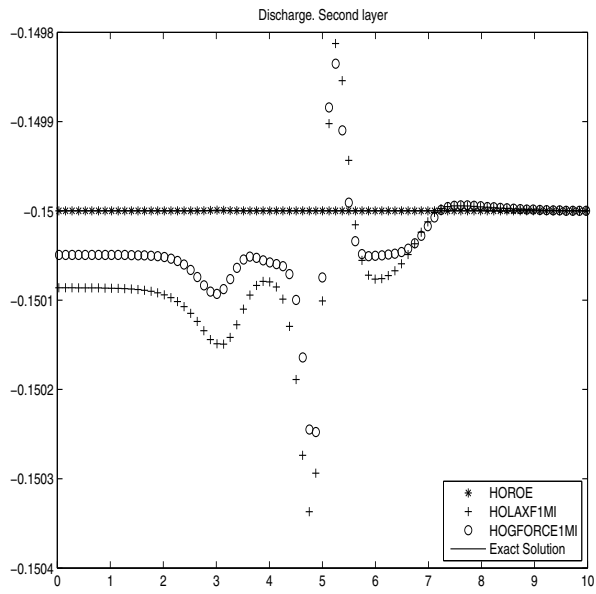

(d) Discharge. Second layer

Figure 7. Test 3: HOROE, HOLAXF1MI and HOGFORCE1MI schemes. Comparison with the exact solution with $\Delta x=1 / 16$.

extensions. This estimate is given by:

$$
\lambda_{\max } \approx\left|\frac{q_{1}+q_{2}}{h_{1}+h_{2}}\right|+\sqrt{g\left(h_{1}+h_{2}\right)}
$$

(see 24] for details).

Observe that ROE and HOROE numerical schemes are more accurate than the others: in particular the accuracy obtained for the discharges of both layers is much better. Due to this, these methods may be preferable when the well-balancing is an important issue. Moreover, in the case of stationary solutions, the computational effort required to achieve a prescribed accuracy is similar for the different numerical schemes: see Figure 8 in which the CPU time is plotted vs. the approximation error in log scale for a fixed mesh composed by 80 cells. As will be seen in Test 6 , this is not the case for time-dependent solutions. 


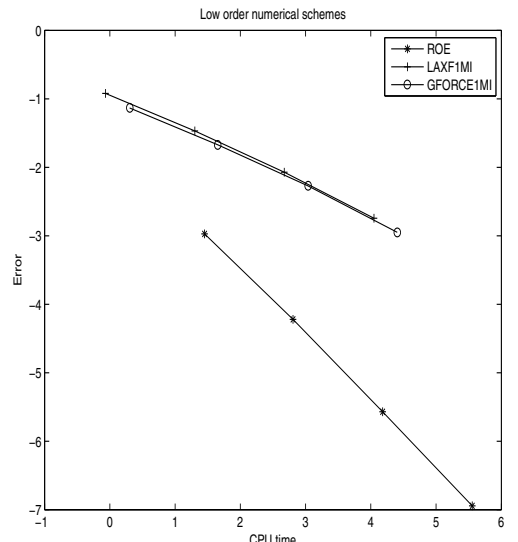

(a) CPU time vs. Error. Low order numerical schemes



(b) CPU time vs. Error. High order numerical schemes

Figure 8. Test 3: CPU time vs. Error (log scale)

5.6. Test 4: Two-layer shallow water. Well-balanced property for a nonsmooth solution. In this test the axis of the channel is the interval $[0,10]$. The bottom topography is given by the function

$$
H(x)=1.0-0.47 e^{-(x-5.0)^{2}} .
$$

The initial condition is $q_{1}(x, 0)=q_{2}(x, 0)=0$, and

$$
h_{1}(x, 0)= \begin{cases}0.5 & \text { if } x<5 \\ 0.03 & \text { otherwise }\end{cases}
$$

and

$$
h_{2}(x, 0)= \begin{cases}0.5-0.47 e^{-(x-5)^{2}} & \text { if } x<5, \\ 0.97-0.47 e^{-(x-5)^{2}} & \text { otherwise. }\end{cases}
$$

As boundary conditions, the relation $q_{1}(\cdot, t)=-q_{2}(\cdot, t)$ is imposed at both ends, and the free surface is fixed to $z=0$ at $x=10$, that is, $h_{1}(10, t)+h_{2}(10, t)-H(x)=0$. The CFL parameter is set to 0.8 and $r=0.98$.

A reference solution is computed with a mesh of 3200 points. Figure 9 shows the comparison of the numerical solutions obtained by ROE, LAXF1MI and GFORCE1MI with the reference solution at time $t=300$ taking $\Delta x=1 / 20$. Figure 10 shows the comparison of the numerical solutions obtained by the high order schemes HOROE, HOLAXF1MI and HOGFORCE1MI with the reference solution at the same time, again taking $\Delta x=1 / 20$.

5.7. Test 5. Order of accuracy: one-layer system. This test is designed to evaluate the accuracy of the schemes for regular time-dependent solutions. The axis of the channel is the interval $[0,10]$ and the bottom function is given by $H(x)=$ 


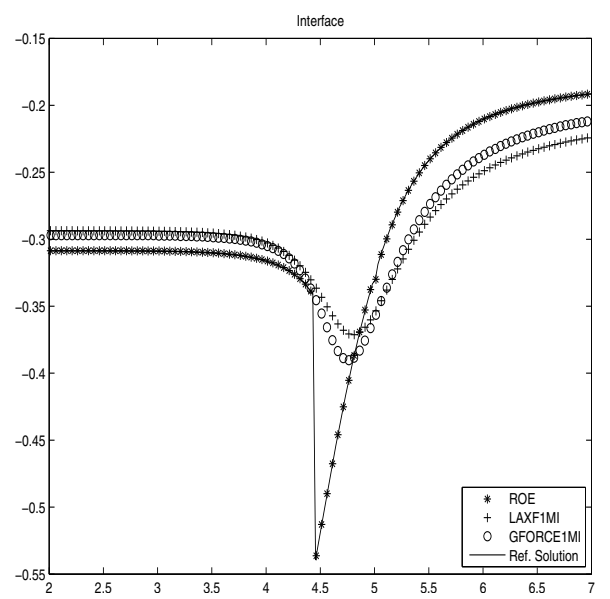

(a) Interface (zoom)

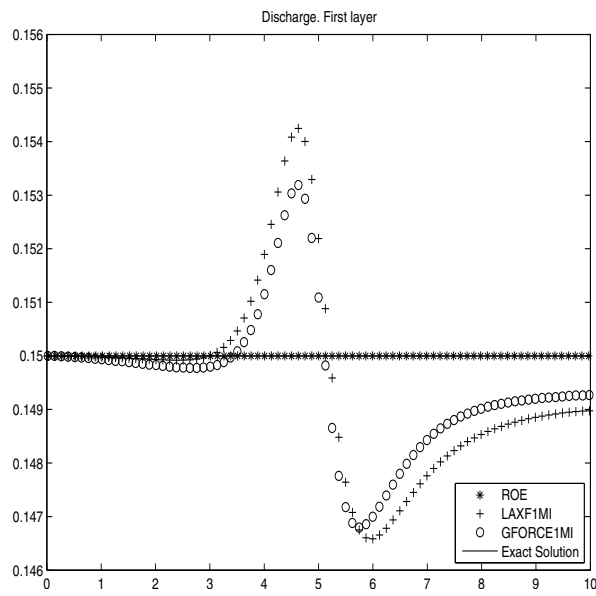

(b) Discharge (zoom). First layer

Figure 9. Test 4: ROE, LAXF1MI and GFORCE1MI schemes. Comparison with the reference solution at time $t=300$ with $\Delta x=$ $1 / 20$.

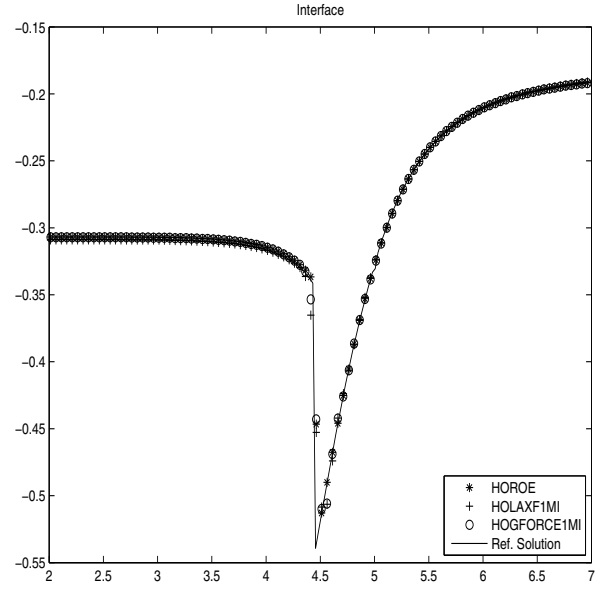

(a) Interface (zoom)

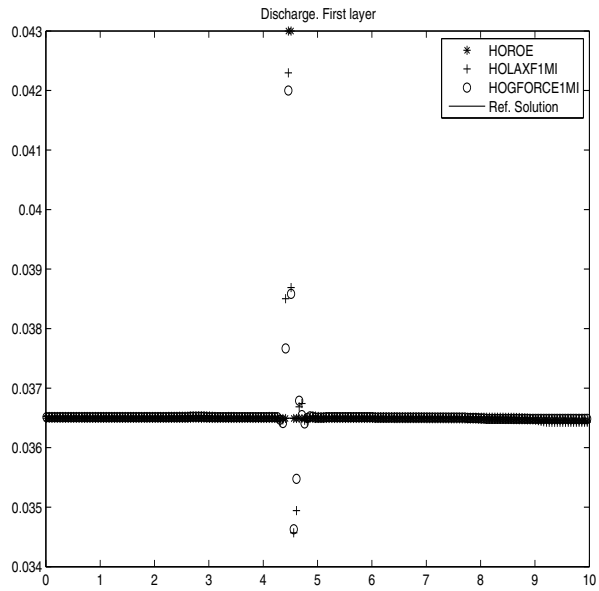

(b) Discharge (zoom). First layer

Figure 10. Test 4: HOROE, HOLAXF1MI and HOGFORCE1MI schemes. Comparison with the reference solution at time $t=300$ with $\Delta x=1 / 20$.

$1-0.5 e^{-3(x-5)^{2}} \sin ^{2}\left(\frac{\pi x}{10}\right)$. The initial condition is $q(x, 0)=0$ and

$$
h(x, 0)= \begin{cases}H(x)+2-0.3\left(1-e^{-0.5(x-5)^{2}}\right), & \text { if } x \geq 5 ; \\ H(x)+2, & \text { if } x<5 .\end{cases}
$$


The final time is $t=0.1$. Periodic boundary conditions are considered. The CFL parameter is set to 0.7 .

A reference solution is computed with a mesh of 20480 points. Table 7 shows the $L^{1}$ errors and the order obtained with the numerical schemes: ROE, LAXF1MI, GFORCE1MI, and their high order extensions HOROE, HOLAXF1MI and HOGFORCE1MI, for five regular meshes with increasing number of cells. As expected, all the numerical schemes achieve first order approximation, ROE being the most accurate, while their high order extensions achieve third order accuracy. It can also be observed that the errors corresponding to HOROE, HOLAXF1MI and HOGFORCE1MI are of the same order.

\begin{tabular}{|c|c|c|c|c|c|c|c|c|}
\hline \multirow[b]{3}{*}{ ells } & \multicolumn{4}{|c|}{$\mathrm{ROE}$} & \multicolumn{4}{|c|}{ HOROE } \\
\hline & \multicolumn{2}{|c|}{$L_{1} h$} & \multicolumn{2}{|c|}{$L_{1} q$} & \multicolumn{2}{|c|}{$L_{1} h$} & \multicolumn{2}{|c|}{$L_{1} q$} \\
\hline & error & order & error & order & error & order & error & order \\
\hline$\overline{80}$ & $2.46 E^{-03}$ & - & $1.39 E^{-03}$ & - & $2.62 E^{-04}$ & & $1.31 E^{-03}$ & - \\
\hline 160 & $6.47 E^{-04}$ & 1.93 & $3.83 E^{-04}$ & 1.86 & $4.47 E^{-05}$ & 2.55 & $2.22 E^{-04}$ & 2.56 \\
\hline 320 & $2.12 E^{-04}$ & 1.63 & $2.17 E^{-04}$ & 0.82 & $7.33 E^{-06}$ & 2.61 & $3.59 E^{-05}$ & 2.63 \\
\hline 640 & $7.78 E^{-05}$ & 1.45 & $1.10 E^{-04}$ & 0.98 & $1.10 E^{-06}$ & 2.73 & $5.08 E^{-06}$ & 2.82 \\
\hline \multirow[t]{3}{*}{1280} & $3.00 E^{-05}$ & 1.37 & $5.00 E^{-05}$ & 1.14 & $1.48 E^{-07}$ & 2.90 & $6.85 E^{-07}$ & 2.89 \\
\hline & \multicolumn{4}{|c|}{ LAXF1MI } & \multicolumn{4}{|c|}{ HOLAXF1MI } \\
\hline & \multicolumn{2}{|c|}{$L_{1} h$} & \multicolumn{2}{|c|}{$L_{1} q$} & \multicolumn{2}{|c|}{$L_{1} h$} & \multicolumn{2}{|c|}{$L_{1} q$} \\
\hline Cells & error & order & error & order & error & order & error & order \\
\hline$\overline{80}$ & 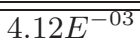 & - & $3.79 E^{-03}$ & - & $3.75 E^{-04}$ & - & $1.70 E^{-03}$ & - \\
\hline 160 & $9.13 E^{-04}$ & 2.17 & $8.45 E^{-04}$ & 2.17 & $6.13 E^{-05}$ & 2.62 & $2.97 E^{-04}$ & 2.52 \\
\hline 320 & $3.55 E^{-04}$ & 1.36 & $5.09 E^{-04}$ & 0.73 & $9.83 E^{-06}$ & 2.64 & $5.00 E^{-05}$ & 2.57 \\
\hline 640 & $1.54 E^{-04}$ & 1.20 & $2.70 E^{-04}$ & 0.92 & $1.47 E^{-06}$ & 2.74 & $7.33 E^{-06}$ & 2.77 \\
\hline \multirow[t]{3}{*}{1280} & $6.95 E^{-05}$ & 1.15 & $1.34 E^{-04}$ & 1.01 & $2.04 E^{-07}$ & 2.85 & $1.02 E^{-06}$ & 2.84 \\
\hline & \multicolumn{4}{|c|}{ GFORCE1MI } & \multicolumn{4}{|c|}{ HOGFORCE1MI } \\
\hline & \multicolumn{2}{|c|}{$L_{1} h$} & \multicolumn{2}{|c|}{$L_{1} q$} & \multicolumn{2}{|c|}{$L_{1} h$} & \multicolumn{2}{|c|}{$L_{1} q$} \\
\hline Cells & error & order & error & order & error & order & error & order \\
\hline 80 & $2.81 E^{-03}$ & - & $2.00 E^{-03}$ & - & $2.88 E^{-04}$ & - & $1.40 E^{-03}$ & - \\
\hline 160 & $6.58 E^{-04}$ & 2.10 & $9.00 E^{-04}$ & 1.69 & $4.53 E^{-0}$ & 2.67 & $2.44 E^{-04}$ & 2.52 \\
\hline 320 & $2.19 E^{-04}$ & 1.59 & $4.74 E^{-04}$ & 0.93 & $6.87 E^{-06}$ & 2.72 & $3.91 E^{-05}$ & 2.64 \\
\hline 640 & $8.25 E^{-05}$ & 1.41 & $1.22 E^{-04}$ & 0.94 & $9.73 E^{-07}$ & 2.82 & $5.69 E^{-06}$ & 2.78 \\
\hline 128 & $3.40 E^{-05}$ & 1.28 & $5.90 E^{-05}$ & 1.05 & $1.30 E^{-07}$ & 2.90 & $7.78 E^{-07}$ & 2.87 \\
\hline
\end{tabular}

TABLE 7. Test 5: Errors and order at $t=0.1$.

5.8. Test 6. Order of accuracy: two-layer system. This test is designed to evaluate the accuracy of the schemes for smooth time-dependent solutions of the homogeneous two-layer shallow water system. We consider a flat channel whose axis is the interval $[-10,10]$. The initial condition is the following:

$$
\begin{array}{ll}
h_{1}(x, 0)=2-e^{-2 x^{2}}(\cos (\pi x / 8) \sin (\pi x / 4))^{2}, & q_{1}(x, 0)=e^{-4 x^{2}}(\sin (\pi x / 4))^{2}, \\
h_{2}(x, 0)=1-e^{-2 x^{2}}(\cos (\pi x / 8) \sin (\pi x / 4))^{2}, & q_{2}(x, 0)=e^{-4 x^{2}}(\sin (\pi x / 2))^{2} .
\end{array}
$$

The final time is $t=1.4$. Periodic boundary conditions are considered. The CFL parameter is set to 0.9 . 
A reference solution is computed with a mesh of 25600 points. Table 8 shows the $L^{1}$ errors (only the maximum error in the four unknowns is shown), the order and the CPU time corresponding to ROE, LAXF1MI, GFORCE1MI, and their high order extensions HOROE, HOLAXF1MI and HOGFORCE1MI, for four regular meshes with increasing number of cells. As expected, all the numerical schemes achieve first order approximation, ROE being the most accurate, while their high order extensions achieve third order accuracy. It can also be observed that the errors corresponding to HOROE, HOLAXF1MI and HOGFORCE1MI are of the same order. Figure 11] shows the CPU time vs. the approximation error in log scale. It can be observed that the CPU time is reduced up to 4.5 times when using the LAXF1MI or 3.15 times when using GFORCE1MI in comparison with $\mathrm{ROE}$, which gives the best results. The CPU time is reduced up to 2.10 times when using HOLAXF1MI or 1.65 times when using HOGFORCE1MI in comparison with HOROE, as in Test 3. Nevertheless, in this case, the accuracy of the high order numerical schemes is quite similar, while HOLAXF1MI and HOGFORCE1MI are cheaper (see Figure 11).

\begin{tabular}{|c|c|c|c|c|c|c|c|c|c|}
\hline & \multicolumn{3}{|c|}{$\mathrm{ROE}$} & \multicolumn{3}{|c|}{ LAXF1MI } & \multicolumn{3}{|c|}{ GFORCE1MI } \\
\hline Cells & error & order & $\mathrm{CPU}$ & error & order & $\mathrm{CPU}$ & error & order & $\mathrm{CPU}$ \\
\hline 400 & $7.73 E^{-2}$ & - & $1.80 E^{0}$ & $1.31 E^{-1}$ & - & $3.98 E^{-1}$ & $1.22 E^{-1}$ & - & $5.71 E^{-1}$ \\
\hline 800 & $4.53 E^{-2}$ & 0.77 & $7.12 E^{0}$ & $7.62 E^{-2}$ & 0.78 & $1.57 E^{0}$ & $7.13 E^{-2}$ & 0.77 & $2.24 E^{0}$ \\
\hline 1600 & $2.49 E^{-2}$ & 0.86 & $2.88 E^{1}$ & $4.23 E^{-2}$ & 0.85 & $6.26 E^{0}$ & $3.85 E^{-2}$ & 0.89 & $8.89 E^{0}$ \\
\hline \multirow[t]{2}{*}{3200} & $1.31 E^{-2}$ & 0.93 & $1.16 E^{2}$ & $2.25 E^{-2}$ & 0.91 & $2.50 E^{1}$ & $2.03 E^{-2}$ & 0.92 & $3.55 E^{1}$ \\
\hline & \multicolumn{3}{|c|}{ HOROE } & \multicolumn{3}{|c|}{ HOLAXF1MI } & \multicolumn{3}{|c|}{ HOGFORCE1MI } \\
\hline Cells & error & order & $\mathrm{CPU}$ & error & order & $\mathrm{CPU}$ & error & order & CPU \\
\hline 400 & $9.39 E^{-3}$ & - & $1.24 E^{1}$ & $1.04 E^{-2}$ & - & $5.54 E^{0}$ & $9.86 E^{-3}$ & - & $7.52 E^{0}$ \\
\hline 800 & $1.88 E^{-3}$ & 2.32 & $4.89 E^{1}$ & $2.24 E^{-3}$ & 2.22 & $2.20 E^{1}$ & $2.09 E^{-3}$ & 2.24 & $2.99 E^{1}$ \\
\hline 1600 & $3.00 E^{-4}$ & 2.65 & $1.95 E^{2}$ & $4.10 E^{-4}$ & 2.45 & $8.71 E^{1}$ & $3.66 E^{-4}$ & 2.51 & $1.19 E^{2}$ \\
\hline 3200 & $3.80 E^{-5}$ & 2.98 & $7.79 E^{2}$ & $5.53 E^{-5}$ & 2.89 & $3.48 E^{2}$ & $4.84 E^{-5}$ & 2.92 & $4.73 E^{2}$ \\
\hline
\end{tabular}

TABLE 8. Test 6 : Errors, order and CPU time at $t=1.4$.

5.9. Test 7: Propagation of perturbations. In order to test the performances of the schemes on a rapidly varying flow over a smooth bed, we consider a test proposed by LeVeque in [17. Specifically, a steady state solution is perturbed by a pulse that splits into two waves propagating in opposite directions over a continuous bed. The left-going wave travels over a horizontal bottom while the right-going wave propagates over a bump. The axis of the channel is the interval $[0,2]$. Outflow boundary conditions have been considered. The bottom topography is given by the function

$$
H(x)= \begin{cases}1-0.25(\cos (10 \pi(x-0.5))+1), & \text { if } 1.4<x<1.6 \\ 1, & \text { otherwise }\end{cases}
$$

The initial data is $q(x, 0)=0$, and

$$
h(x, 0)= \begin{cases}H(x)+\Delta h, & \text { if } 1.1<x<1.2 \\ H(x), & \text { otherwise }\end{cases}
$$




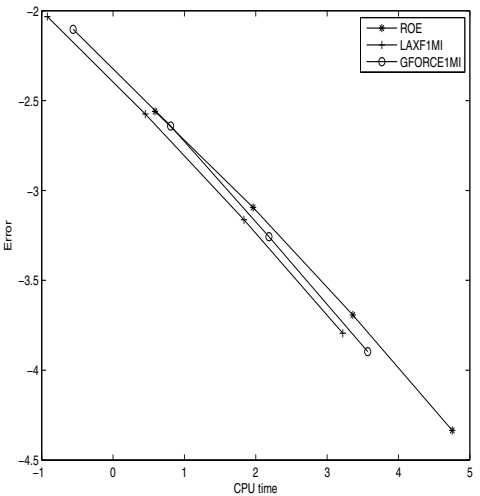

(a) CPU time vs. Error. Low order numerical schemes

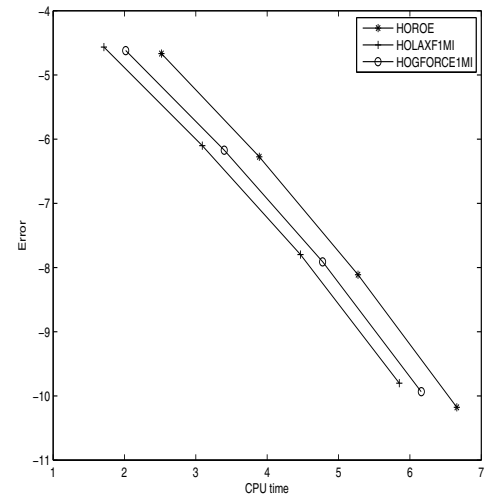

(b) CPU time vs. Error. High order numerical schemes

Figure 11. Test 6: CPU time vs. Error (log scale)

Here $\Delta h$ is the height of the perturbation that takes the value $\Delta h=0.001$ (small pulse) (see Figure 12). Free boundary conditions are imposed and the CFL parameter is set to 0.9 .

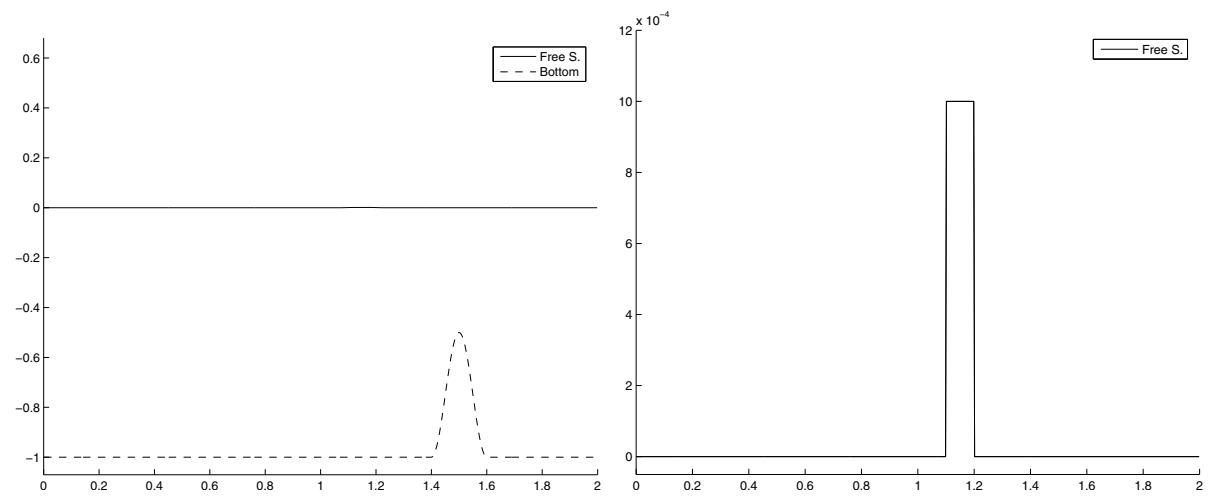

Figure 12. Test 7: Perturbed steady state.

A reference solution is computed with a mesh of 20480 cells. In Figure 13 we compare the results obtained with ROE, LAXF1MI, GFORCE1MI, and the reference solution at time $t=0.2$ taking $\Delta x=1 / 40$. Figure 14 corresponds to the solutions obtained with the high order schemes at the same time using the same mesh. Notice that, while the conclusions for first order schemes are similar to those of the previous test cases, for high order schemes the differences are very small.

5.10. Test 8: Wet/dry fronts in a nonflat basin. In order to test the performances of the schemes in the presence of wet/dry zones, we consider a problem proposed by Gallouët et al. in [13, specifically designed to assess the behavior on 


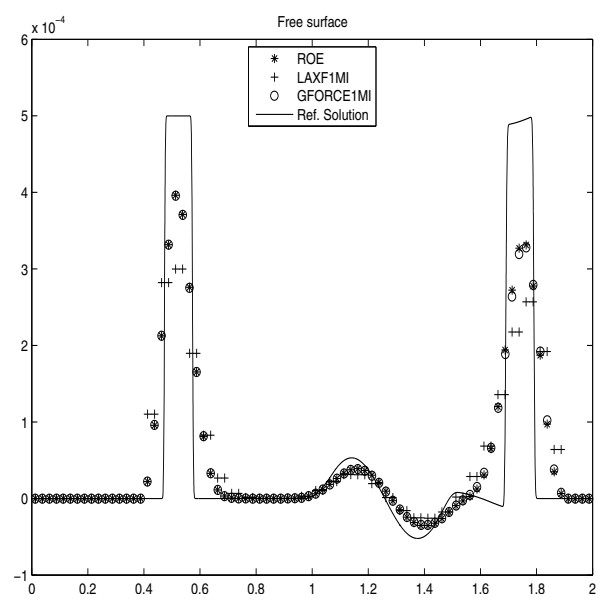

(a) Free surface.

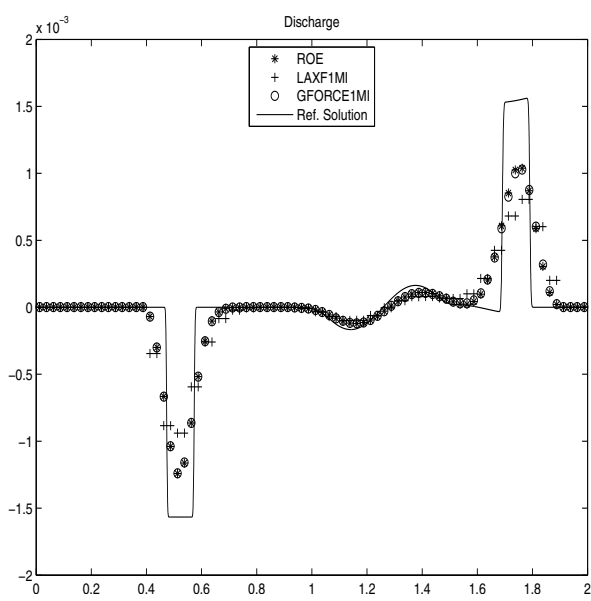

(b) Discharge.

Figure 13. Test 7: First order numerical schemes. Comparison with the reference solution at $t=0.2$ with $\Delta x=1 / 40$.

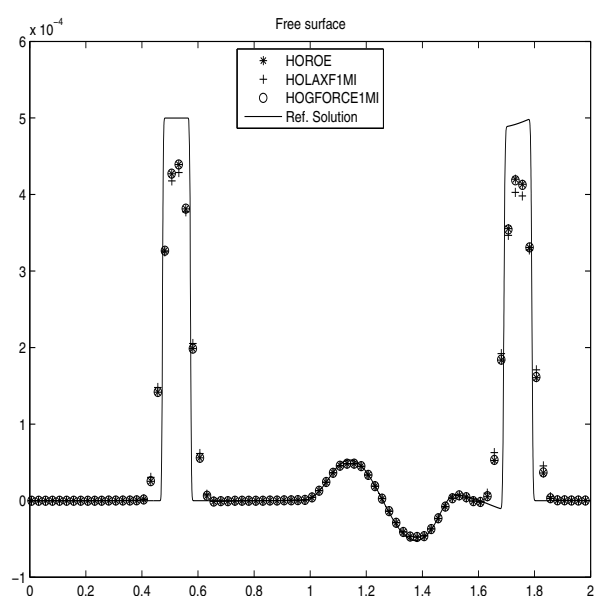

(a) Free surface.

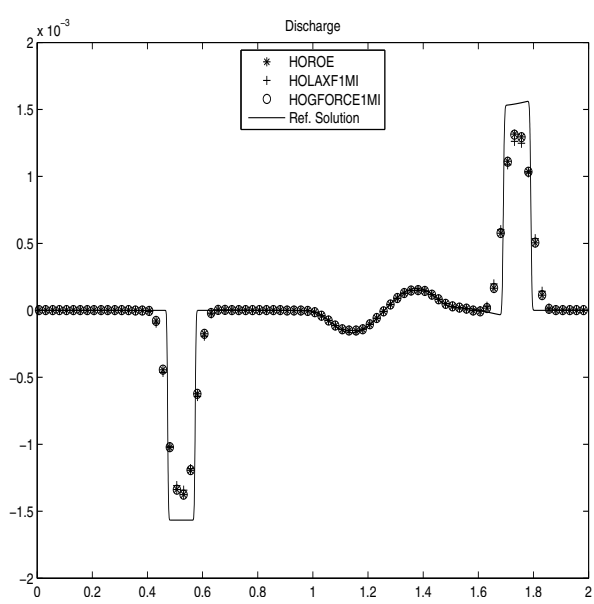

(b) Discharge.

Figure 14. Test 7: High order numerical schemes. Comparison with the reference solution at $t=0.2$ with $\Delta x=1 / 40$.

drying a nonflat basin. In this test case, a dry bed is formed in the middle of two rarefaction waves traveling in opposite directions. The generation of the dry bed makes this problem numerically difficult, especially when the left-going rarefaction wave propagates over the step. 
The axis of the channel is the interval $[0,25]$. The bottom topography is given by the function

$$
H(x)= \begin{cases}13, & \text { if } 25 / 3<x<25 / 2 \\ 14, & \text { otherwise. }\end{cases}
$$

The initial condition is

$$
q(x, 0)= \begin{cases}-300, & \text { if } \frac{50}{3} \leq x, \\ 300, & \text { if } \frac{50}{3}>x,\end{cases}
$$

and $h(x, 0)=H(x)-4$.

A reference solution is computed using the Roe scheme with a mesh of 20480 points. Figure [15] shows the comparison of the numerical results obtained with ROE, LAXF1MI, and GFORCE1MI, and the reference solution at time $t=0.25$ taking $\Delta x=5 / 32$. Figure 16 corresponds to the comparison of their high order extension at the same time using the same mesh. As can be seen, all the numerical schemes capture correctly the generation and propagation of the wet-dry fronts. The conclusions are similar to those of the previous test case.

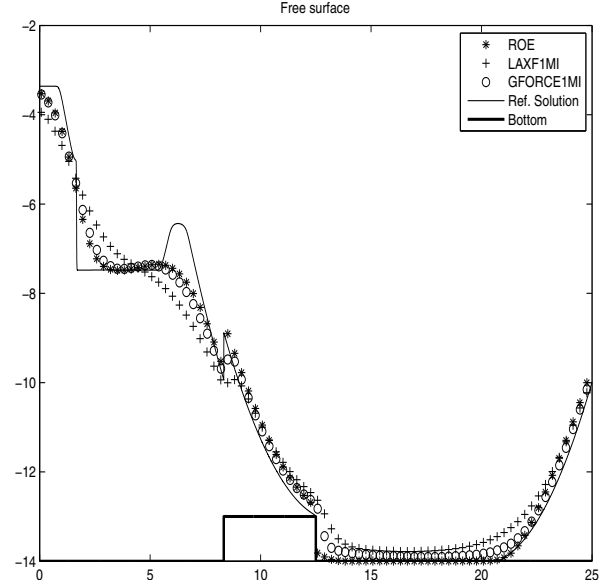

(a) Free surface.

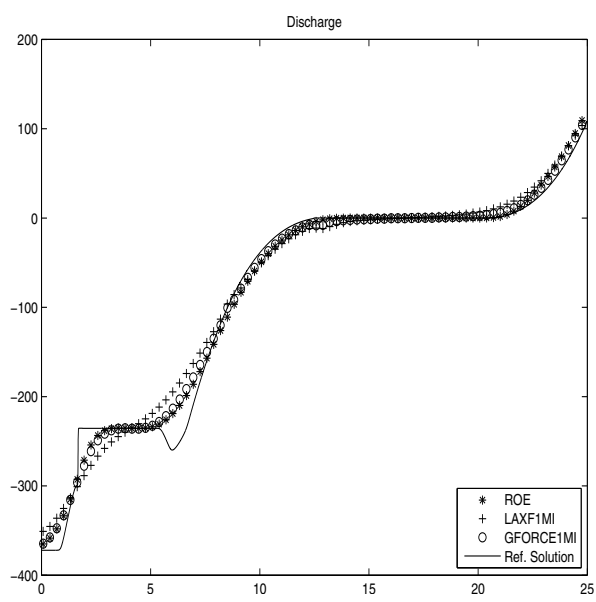

(b) Discharge.

Figure 15. Test 8: First order numerical schemes. Comparison with the reference solution at $t=0.25$ with $\Delta x=5 / 32$.

5.11. Test 9: Internal dam-break problem. This test is designed to evaluate the accuracy of the schemes for nonregular time-dependent solutions over a flat bottom. The axis of the channel is the interval $[0,10]$.

The initial condition is $q_{1}(x, 0)=q_{2}(x, 0)=0$,

$$
h_{1}(x, 0)= \begin{cases}0.6, & \text { if } x<5, \\ 0.4, & \text { otherwise }\end{cases}
$$




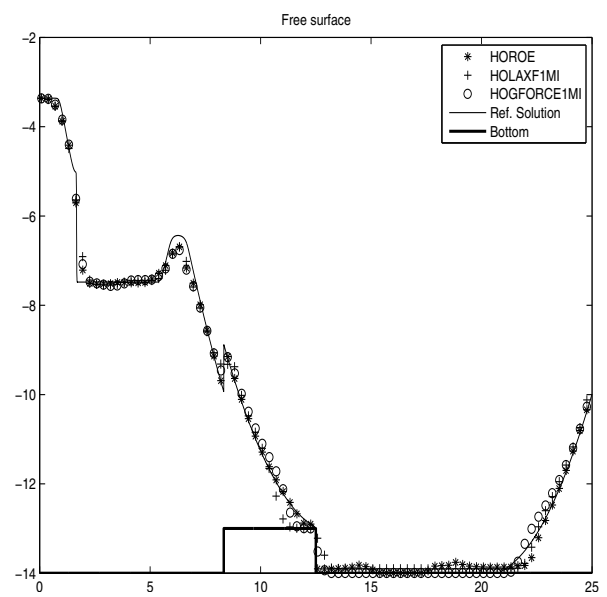

(a) Free surface.

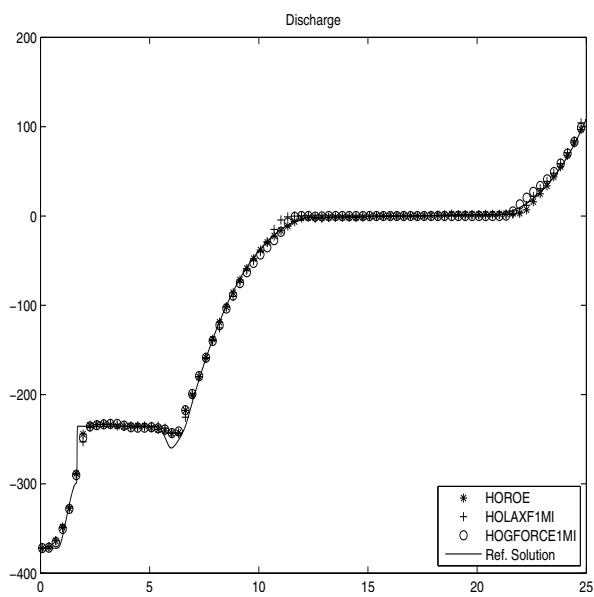

(b) Discharge.

Figure 16. Test 8: High order numerical schemes. Comparison with the reference solution at $t=0.25$ with $\Delta x=5 / 32$.

and

$$
h_{2}(x, 0)= \begin{cases}0.4, & \text { if } x<5, \\ 0.6, & \text { otherwise. }\end{cases}
$$

Free boundary conditions are considered. The CFL parameter is set to 0.9 and $r=0.98$.

A reference solution is computed with a mesh of 3200 points.

Figures 17 and 18 show the comparison of the numerical results of both first and high order numerical schemes with the reference solution at time $t=10 \mathrm{~s}$ taking $\Delta x=1 / 20$.

Finally, as in the previous numerical tests, the differences between the high order numerical schemes are much smaller than they are for first order numerical schemes (see Figure 17).

The numerical results of this test seem to show that the numerical solutions provided by the different numerical schemes converge to the same solution, but this is not the case: there are slight differences between the speed and the limit states of the shocks for the limits of the solutions obtained with the different schemes as $\Delta x$ tends to 0 . Nevertheless, a finer analysis is required to notice this fact. This is done in the next test case.

5.12. Test 10: Two-layer shallow-water system. Approximation of internal shocks. This test is specifically designed to show that, in the presence of shocks, the numerical solutions provided by the different numerical schemes converge to different weak solutions of the homogeneous two-layer shallow-water system, even if all of them are based on the same Roe linearization and thus on the same family of paths (the straight segments in this case). We consider here Roe, Lax-Friedrichs and GFORCE methods. 


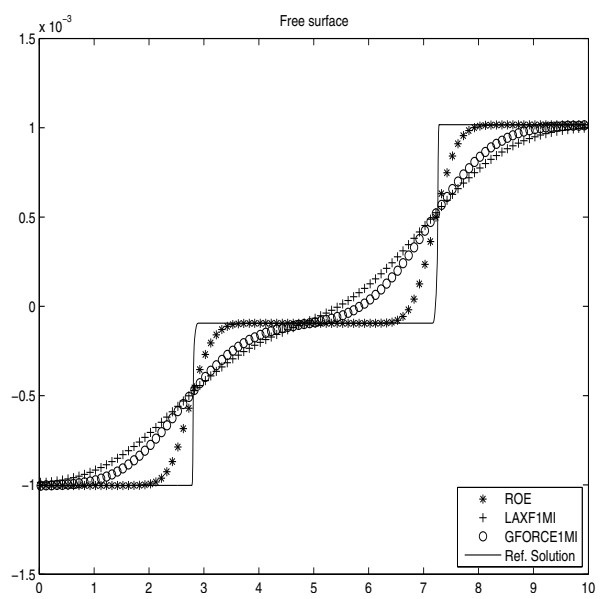

(a) Free Surface.

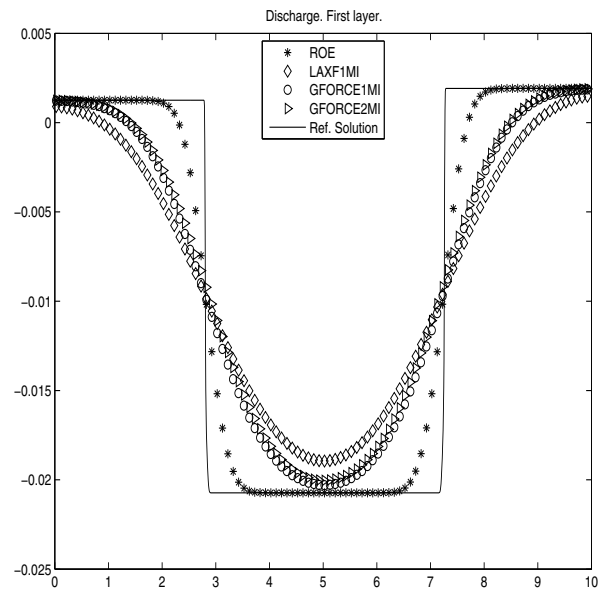

(c) Discharge. First layer.

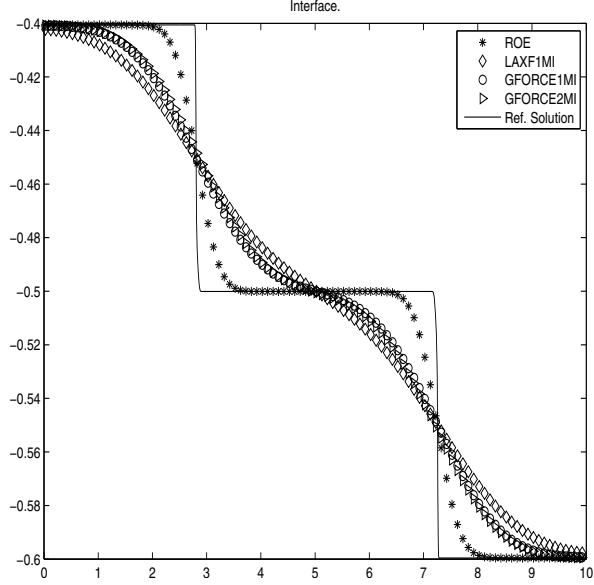

(b) Interface. First layer

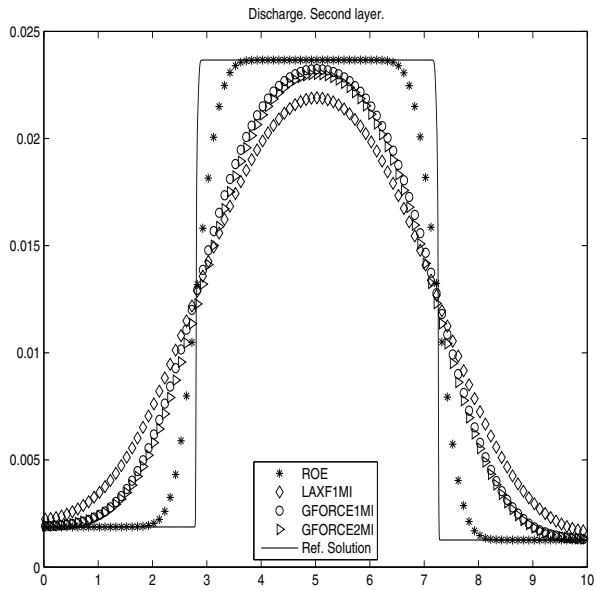

(d) Discharge. Second layer

Figure 17. Test 9: ROE, LAXF1MI, and GFORCE1MI schemes. Comparison with the reference solution at $t=10 \mathrm{~s}$ with $\Delta x=$ $1 / 20$.

When the family of straight segments is also used to define the weak solutions of the system, the Rankine-Hugoniot conditions satisfied at a jump are the following:

$$
\left\{\begin{array}{l}
\xi\left(h_{1}^{r}-h_{1}^{l}\right)=q_{1}^{r}-q_{1}^{l} ; \\
\xi\left(q_{1}^{r}-q_{1}^{l}\right)=\frac{\left(q_{1}^{r}\right)^{2}}{h_{1}^{r}}-\frac{\left(q_{1}^{l}\right)^{2}}{h_{1}^{l}}+\frac{g}{2}\left(h_{1}^{r}\right)^{2}-\frac{g}{2}\left(h_{1}^{l}\right)^{2}+g \frac{h_{1}^{l}+h_{1}^{r}}{2}\left(h_{2}^{r}-h_{2}^{l}\right) ; \\
\xi\left(h_{2}^{r}-h_{2}^{l}\right)=q_{2}^{r}-q_{2}^{l} ; \\
\xi\left(q_{2}^{r}-q_{2}^{l}\right)=\frac{\left(q_{2}^{r}\right)^{2}}{h_{2}^{r}}-\frac{\left(q_{2}^{l}\right)^{2}}{h_{2}^{l}}+\frac{g}{2}\left(h_{2}^{r}\right)^{2}-\frac{g}{2}\left(h_{2}^{l}\right)^{2}+g r \frac{h_{2}^{l}+h_{2}^{r}}{2}\left(h_{1}^{r}-h_{1}^{l}\right),
\end{array}\right.
$$




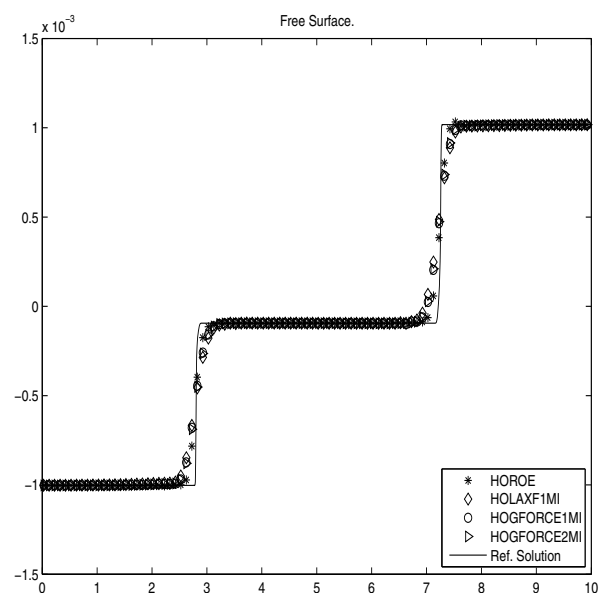

(a) Free Surface.

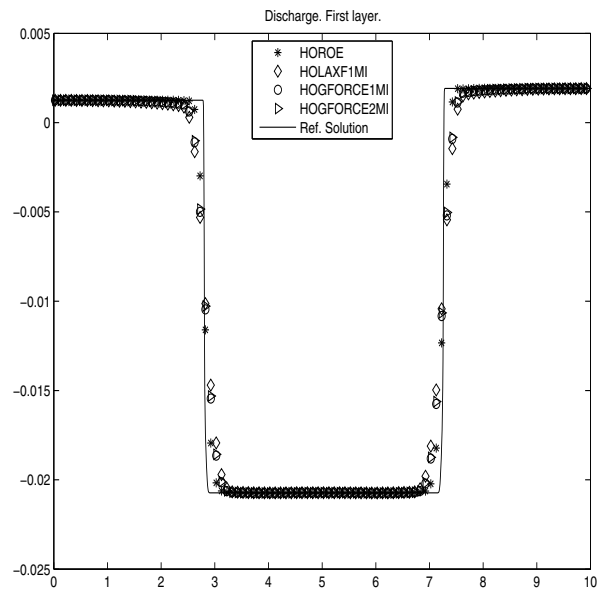

(c) Discharge. First layer.

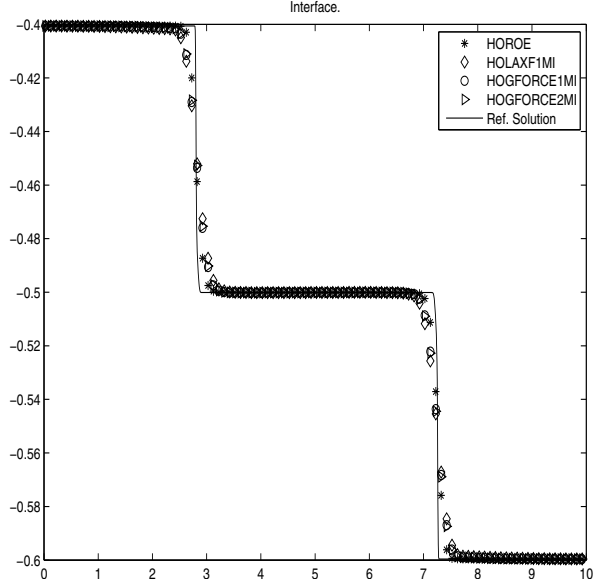

(b) Interface. First layer

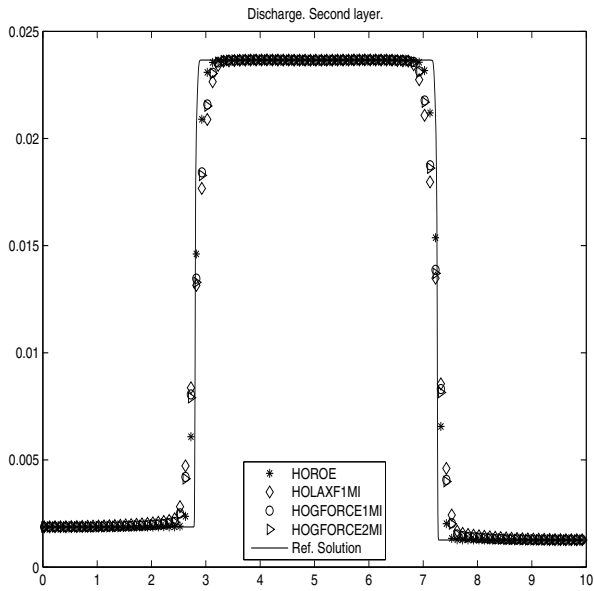

(d) Discharge. Second layer

Figure 18. Test 9: HOROE, HOLAXF1MI, and HOGFORCE1MI schemes. Comparison with the reference solution at $t=10 \mathrm{~s}$ with $\Delta x=1 / 20$.

where $\xi$ is the speed of propagation of the shock and

$$
w_{l}=\left[\begin{array}{c}
h_{1}^{l} \\
q_{1}^{l} \\
h_{2}^{l} \\
q_{2}^{l}
\end{array}\right], \quad w_{r}=\left[\begin{array}{c}
h_{1}^{r} \\
q_{1}^{r} \\
h_{2}^{r} \\
q_{2}^{r}
\end{array}\right]
$$

are the values to the left and to the right, respectively.

In all of the cases considered here the order of the eigenvalues of the system is:

$$
\lambda_{\text {ext }}^{-}<\lambda_{\text {int }}^{-}<\lambda_{\text {int }}^{+}<\lambda_{\text {ext }}^{+} \text {. }
$$

Moreover

$$
\left|\lambda_{\text {int }}^{ \pm}\right| \ll\left|\lambda_{\text {ext }}^{ \pm}\right| .
$$




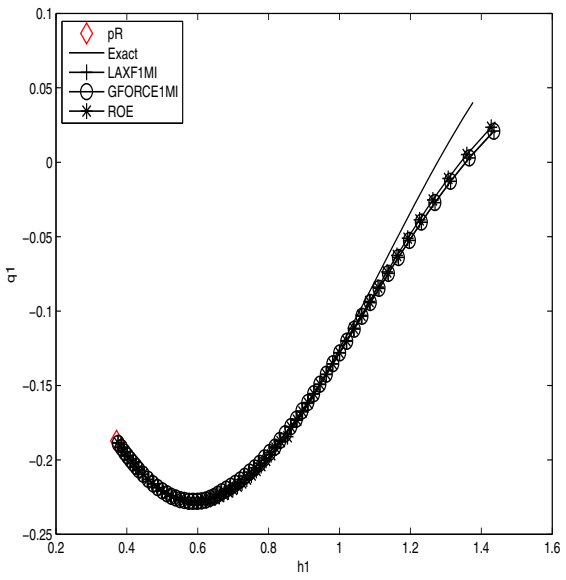

(a) Hugoniot curves (plane $\left.\left(h_{1}, q_{1}\right)\right)$

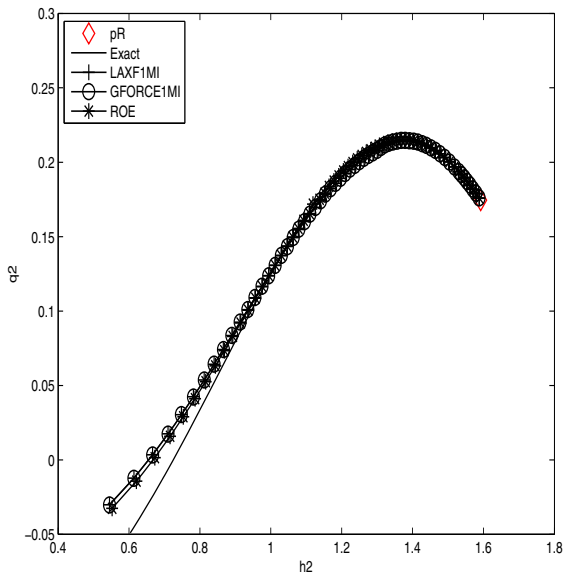

(b) Hugoniot curves (plane $\left(h_{2}, q_{2}\right)$ )

Figure 19. Test 10: Hugoniot curves: exact (continuous line) and numerical (lines with dots).

The goal here is to compare the exact and the numerical Hugoniot curves corresponding to one of the internal characteristic fields, i.e., the fields related to the eigenvalues $\lambda_{\text {int }}^{ \pm}$. We proceed as follows: the state

$$
w_{r}=\left[\begin{array}{c}
h_{1}^{r} \\
q_{1}^{r} \\
h_{2}^{r} \\
q_{2}^{r}
\end{array}\right]=\left[\begin{array}{c}
0.392034161025472 \\
-0.198826959396196 \\
1.588829011097482 \\
0.186046955388750
\end{array}\right]
$$

is fixed. Then, we compute the Hugoniot curve corresponding to the "left" states $w_{l}$ that can be connected with $w_{r}$ with a 3 -shock with $r=0.98$. To do this, we use the speed of the shock $\xi$ as a parameter and, for each value of $\xi$ we solve the nonlinear system (5.14). In Figure 19 we show the projection (continuous line) of the computed Hugoniot curve onto the planes $\left(h_{1}, q_{1}\right)$ (left) and $\left(h_{2}, q_{2}\right)$ (right), respectively.

Next, we consider a family of Riemann problems whose states are $w_{r}$, while $w_{l}$ runs on the Hugoniot curve. While the exact solution of the Riemann problem consists only of an internal shock, this is not the case for the numerical solutions. We have solved this family of Riemann problems using Roe, Lax-Friedrichs, and GFORCE. The first divided difference is used then as a smoothness indicator to determine the speed of propagation and the limit states of the shock corresponding to the eigenvalue $\lambda_{i n t}^{+}$in the numerical solutions. These calculations have been performed by using four meshes with decreasing steps $(\Delta x=0.002,0.001,0.0005$ and 0.00025). We check that all the numerical schemes converge, but their limits do not satisfy the Rankine-Hugoniot conditions (5.14). The numerical Hugoniot curves corresponding to $\Delta x=0.001$ are compared with the exact one in Figure 19. 
Notice that, if $w_{r}$ and $w_{l}$ are close enough, all the curves are very close. The same behaviour can be observed when the speed of the shock is close to zero.

\section{Conclusions}

Two families of first order path-conservative numerical schemes for nonconservative systems have been introduced in this article, which consists of a convex combination of a generalized Lax-Friedrichs scheme and a generalized Lax-Wendroff scheme. While in the first family the generalization of the Lax-Wendroff scheme is first order accurate, in the second family it is second order accurate for general problems. All of these schemes are based on a Roe linearization of the system and among them, there are extensions of the FORCE and GFORCE conservative methods. Well-balanced versions of these schemes for solving systems of conservation laws with source terms and/or nonconservative products have also been introduced, together with some modifications to take into account the difficulty related to resonance.

The numerical schemes have been extended to high order by following the ideas developed in 17, 20, 12, by means of the use of the PHM third order reconstruction operator (see [18]).

Concerning the quality of the numerical solutions and the well-balanced properties, the best first order numerical scheme is Roe, followed by GFORCE. The results obtained by FORCE schemes are very close to those obtained by GFORCE. Although Rusanov and Lax-Friedrichs schemes are more diffusive, these methods present the advantage of having good entropy and positivity-preserving properties for homogeneous problems. These properties may be preserved for problems with a source-term if a convenient generalized hydrostatic reconstruction is used.

The high order extensions are similar regardless of the first order scheme considered. Nevertheless, when the analytical expression of the eigenvalues and eigenvectors of the matrix is not available (as is the case for the two-layer system), the CPU time is reduced if a numerical scheme of the families introduced here is used. Nevertheless, in the case of stationary solutions, due to the improved accuracy of Roe methods for this type of solutions, this reduction of the CPU time is lower: the computational effort to obtain a prescribed accuracy is similar for the different schemes.

As a conclusion, the numerical schemes studied are an excellent alternative to Roe methods to approximate time-dependent solutions when the calculation of the eigenvalues has to be numerically performed and the schemes are going to be used as the basis for higher order methods or multidimensional problems.

Appendix A. As in [19] we will assume here that the family of paths fulfills the following requirements:

(R1) Given an integral curve $\gamma$ of a linearly degenerate field and $W_{L}, W_{R} \in \gamma$, the path $\Phi\left(s ; W_{L}, W_{R}\right)$ is a parameterization of the arc of $\gamma$ linking $W_{L}$ and $W_{R}$.

(R2) Given an integral curve $\gamma$ of a genuinely nonlinear field $R_{i}$ and $W_{L}, W_{R} \in \gamma$, with $\lambda_{i}\left(W_{L}\right)<\lambda_{i}\left(W_{R}\right)$, the path $\Phi\left(s ; W_{L}, W_{R}\right)$ is a parameterization of the arc of $\gamma$ linking $W_{L}$ and $W_{R}$. 
(R3) Let us denote by $\mathcal{R P} \subset \Omega \times \Omega$ the set of pairs $\left(W_{L}, W_{R}\right)$ for which the Riemann problem

$$
\left\{\begin{array}{l}
W_{t}+\mathcal{A}(W) W_{x}=0, \\
W(x, 0)= \begin{cases}W_{L} & \text { if } x<0, \\
W_{R} & \text { if } x>0\end{cases}
\end{array}\right.
$$

has a unique self-similar weak solution composed of at most $N$ simple waves connecting $J+1$ intermediate constant states

$$
W_{0}=W_{L}, W_{1}, \ldots, W_{J-1}, W_{J}=W_{R},
$$

with $J \leq N$. Then, given $\left(W_{L}, W_{R}\right) \in \mathcal{R P}$, the curve described by the path $\Phi\left(\cdot ; W_{L}, W_{R}\right)$ is equal to the union of those corresponding to the paths $\Phi\left(\cdot ; W_{j-1}, W_{j}\right), j=1, \ldots, J$.

Proof of Proposition 3.1. Let us consider the family of Riemann problems:

$$
\left\{\begin{array}{l}
W_{t}+\frac{1}{2}(\mathcal{A}(W)+\beta \cdot I d) W_{x}=0 \\
W(x, 0)= \begin{cases}W_{L} & \text { if } x<0 \\
W_{R} & \text { if } x>0\end{cases}
\end{array}\right.
$$

where the family of paths $\Phi$ is used to give a sense to the nonconservative products. It can be easily verified that the function

$$
V_{\beta}\left(s ; W_{L}, W_{R}\right)=V\left(2 s-\beta ; W_{L}, W_{R}\right),
$$

where $V\left(x / t ; W_{L}, W_{R}\right)$ denotes the self-similar entropy solution of the Riemann problem (6.1), is a self-similar solution of (6.2). Moreover, it consists of the same intermediate states appearing at the solution of (6.1) and the simple waves linking them are of the same nature but their velocites are averaged with $\beta$. Moreover, it is an entropy solution for the pair $\left(\eta, G_{\beta}\right)$, given by

$$
G_{\beta}(W)=\frac{1}{2}(G(W)+\beta \eta(W)) .
$$

The following equality is thus satisfied:

$$
\begin{aligned}
& G_{\beta}\left(W_{R}\right)+\int_{0}^{\infty}\left(\eta\left(V_{\beta}\left(v ; W_{L}, W_{R}\right)\right)-\eta\left(W_{R}\right)\right) d v \\
& \leq G_{\beta}\left(W_{L}\right)-\int_{-\infty}^{0}\left(\eta\left(V_{\beta}\left(v ; W_{L}, W_{R}\right)\right)-\eta\left(W_{L}\right)\right) d v .
\end{aligned}
$$

Notice now that, if $\beta$ is positive and the inequality

$$
\left|\lambda_{j}\left(W_{k}\right)\right| \leq \beta, \quad j=1, \ldots, N ; k=0, \ldots, J,
$$

is satisfied, where $W_{0}, \ldots, W_{J}$ are the intermediate states appearing at the solutions of the Riemann problems, then all the speeds of the simple waves involved by the 
solution of (6.2) are positive and thus the following equality holds:

$$
\begin{aligned}
- & \int_{0}^{\infty}\left(V_{\beta}\left(v ; W_{L}, W_{R}\right)-W_{R}\right) d v \\
& =\left\langle\frac{1}{2}\left[(\mathcal{A}(W(\cdot, t))+\beta \cdot I d) W_{x}(\cdot, t)\right]_{\Phi}, 1\right\rangle \\
& =\frac{1}{2} \int_{0}^{1} \mathcal{A}\left(\Phi\left(s ; W_{L}, W_{R}\right)\right) \frac{\partial \Phi}{\partial s}\left(s ; W_{L}, W_{R}\right) d s+\frac{1}{2} \beta\left(W_{R}-W_{L}\right) \\
& =\frac{1}{2}\left(A_{\Phi}\left(W_{L}, W_{R}\right)+\beta \cdot I d\right) \cdot\left(W_{R}-W_{L}\right),
\end{aligned}
$$

where (2.7) and the properties (R1)-(R3) of $\Phi$ have been used (see 19]). In this case, the entropy inequality (6.5) reduces to

$$
G_{\beta}\left(W_{R}\right)+\int_{0}^{\infty}\left(\eta\left(V_{\beta}\left(v ; W_{L}, W_{R}\right)\right)-\eta\left(W_{R}\right)\right) d v \leq G_{\beta}\left(W_{L}\right) .
$$

Using similar arguments we can also obtain

$$
\begin{aligned}
-\int_{-\infty}^{0}\left(V_{-\beta}\left(v ; W_{L}, W_{R}\right)-W_{L}\right) & d v \\
= & \frac{1}{2}\left(A_{\Phi}\left(W_{L}, W_{R}\right)-\beta \cdot I d\right) \cdot\left(W_{R}-W_{L}\right)
\end{aligned}
$$

and

$$
G_{-\beta}\left(W_{R}\right) \leq G_{-\beta}\left(W_{L}\right)-\int_{-\infty}^{0}\left(\eta\left(V_{-\beta}\left(v ; W_{L}, W_{R}\right)\right)-\eta\left(W_{L}\right)\right) d v .
$$

Adding (6.8) and (6.10), we obtain:

$$
\begin{aligned}
G\left(W_{R}\right)+\int_{0}^{\infty}\left(\eta\left(V_{\beta}\left(v ; W_{L}, W_{R}\right)\right)-\eta\left(W_{R}\right)\right) d v & \\
\leq & G\left(W_{L}\right)-\int_{-\infty}^{0}\left(\eta\left(V_{-\beta}\left(v ; W_{L}, W_{R}\right)\right)-\eta\left(W_{L}\right)\right) d v .
\end{aligned}
$$

Taking into account the equalities (6.7), (6.9) with $\beta=\alpha\left(W_{L}, W_{R}\right)$, it can be easily seen that, under the hypotheses (3.8) and (3.9), the generalized Rusanov scheme can be interpreted as the numerical method based on the approximate Riemann solver:

$$
\widetilde{V}\left(s ; W_{L}, W_{R}\right) \begin{cases}V_{-\alpha\left(W_{L}, W_{R}\right)}\left(s ; W_{L}, W_{R}\right) & \text { if } s<0 ; \\ V_{\alpha\left(W_{L}, W_{R}\right)}\left(s ; W_{L}, W_{R}\right) & \text { if } s>0 .\end{cases}
$$

Finally, from (6.11) with $\beta=\alpha\left(W_{L}, W_{R}\right)$, it can be easily deduced that (3.10) holds for any numerical entropy flux function $\widehat{G}\left(W_{L}, W_{R}\right)$ satisfying:

$$
\begin{aligned}
& G\left(W_{R}\right)+\int_{0}^{\infty}\left(\eta\left(V_{\alpha\left(W_{L}, W_{R}\right)}\left(v ; W_{L}, W_{R}\right)\right)-\eta\left(W_{R}\right)\right) d v \\
& \quad \leq \widehat{G}\left(W_{L}, W_{R}\right) \\
& \quad \leq G\left(W_{L}\right)-\int_{-\infty}^{0}\left(\eta\left(V_{-\alpha\left(W_{L}, W_{R}\right)}\left(v ; W_{L}, W_{R}\right)\right)-\eta\left(W_{L}\right)\right) d v .
\end{aligned}
$$


Proof of Proposition 3.2. It has been seen in the proof of Proposition 3.1 that the numerical scheme can be interpreted as the numerical method based on the approximate Riemann solver (6.12). From this representation, the proof is trivial by taking into account the relations (6.3) and (3.11).

\section{REFERENCES}

[1] E. Audusse, F. Bouchut, M.O. Bristeau, R. Klein, B. Perthame. A fast and stable wellbalanced scheme with hydrostatic reconstruction for shallow water flows, SIAM J. Sci. Comp. 25 (6): 2050-2065, 2004. MR2086830 (2005f:76069)

[2] A. Bermúdez, M.E. Vázquez. Upwind methods for hyperbolic conservation laws with source terms. Computers and Fluids, 23(8): 1049-1071, 1994. MR1314237(95i:76065)

[3] F. Bouchut. Nonlinear Stability of Finite Volume Methods for Hyperbolic Conservation Laws and Well-Balanced Schemes for Sources. Birkhäuser, 2004. MR.2128209 (2005m:65002)

[4] A. Canestrelli, A. Siviglia, M. Dumbser, E.F. Toro. Well-balanced high-order centered schemes for non-conservative hyperbolic systems. Applications to Shallow Water Equations with fixed and mobile bed. Adv. Water Resour, doi:10.1016/j.advwatres.2009.02.006, 2009.

[5] M. Castro, A. Ferreiro, J.A. García-Rodriguez, J.M. González, J. Macías, C. Parés, M.E. Vázquez-Cendón. On the numerical treatment of wet/dry fronts in shallow flows: Application to one-layer and two-layer 1-D shallow water system. Math. and Comp. Model., 42: 419-439, 2005. MR2163780 (2006c:76021)

[6] M.J. Castro, P.G. LeFloch, M.L. Muñoz, C. Parés. Why many theories of shock waves are necessary: Convergence error in formally path-consistent schemes. J. Comput. Phys. 227: 8107-8129, 2008. MR2442446 (2009g:76071)

[7] M.J. Castro, J.M Gallardo, C. Parés. High order finite volume schemes based on reconstruction of states for solving hyperbolic systems with nonconservative products. Applications to shallow water systems. Math. Comp., 75: 1103-1134, 2006. MR2219021 (2007a:65119)

[8] M.J. Castro, J. Macías, C. Parés. A Q-Scheme for a class of systems of coupled conservation laws with source term. Application to a two-layer 1-D shallow water system. Math. Mod. Num. Anal. 35(1): 107-127, 2001. MR1811983 (2001m:76063)

[9] M.J. Castro, A. Pardo, C. Parés. Well-balanced numerical schemes based on a generalized hydrostatic reconstruction technique. Math. Mod. Meth. App. Sci., 17: 2055-2113, 2007. MR 2371563 (2009c:65186)

[10] G. Dal Maso, P.G. LeFloch, F. Murat. Definition and weak stability of nonconservative products. J. Math. Pures Appl. 74: 483-548, 1995. MR.1365258 (97b:46052)

[11] B. Einfeldt. On Godunov-type methods for gas dynamics. SIAM J. Numer. Anal. 25: 294318, 1988. MR933726(89e:65086)

[12] J.M. Gallardo, C. Parés, M. Castro. On a well-balanced high-order finite volume scheme for shallow water equations with topography and dry areas. J. Comput. Phys. 227: 574-601, 2007. MR2361537 (2008m:76080)

[13] T. Gallouët, J.M. Hérard, N. Seguin. Some approximate Godunov schemes to compute shallow-water equations with topography. Computers and Fluids 32: 479-513, 2003. MR.1966639(2004a:76095)

[14] A. Harten, J.M. Hyman. Self-adjusting grid methods for one-dimensional hyperbolic conservation laws. J. Comput. Phys. 50: 235-269, 1983. MR707200 (85g:65111)

[15] T.Y. Hou, P.G. LeFloch. Why nonconservative schemes converge to wrong solutions: Error analysis. Math. of Comput. 62: 497-530, 1994. MR1201068 (94g:65093)

[16] P.G. LeFloch, T.P. Liu.Existence theory for nonlinear hyperbolic systems in nonconservative form. Forum Math. 5: 261-280, 1993. MR 1216035 (94e:35086)

[17] R. LeVeque. Balancing source terms and flux gradients in high-resolution Godunov methods: The quasi-steady wave-propagation algorithm. J. Comput. Phys. 146: 346-365, 1998. MR $1650496(99 \mathrm{j}: 65182)$

[18] A. Marquina. Local piecewise hyperbolic reconstructions for nonlinear scalar conservation laws.. SIAM J. Sci. Comp 15: 892-915, 1994. MR1278006 (95a:65137)

[19] M.L. Muñoz, C. Parés. Godunov method for nonconservative hyperbolic systems, ESAIM: M2AN 41(1): 169-185, 2007. MR2323696 (2008g:65112)

[20] C. Parés. Numerical methods for nonconservative hyperbolic systems: A theoretical framework. SIAM J. Num. Anal. 44(1): 300-321, 2006. MR2217384(2006m:65169) 
[21] C. Parés, M.J. Castro. On the well-balance property of Roe's method for nonconservative hyperbolic systems. Applications to Shallow-Water Systems. M2AN, 38(5): 821-852, 2004. MR 2104431 (2006f:65085)

[22] S. Noelle, Y. Xing, C.W. Shu. High-order well-balanced finite volume WENO schemes for shallow water equation with moving water, J. Comput. Phys. 226: 29-58, 2007. MR2356351 (2008f:76124)

[23] P.L. Roe. Approximate Riemann solvers, parameter vectors and difference schemes. J. Comput. Phys. 43: 357-371, 1981. MR640362 (82k:65055)

[24] J.B. Schijf, J.C. Schonfeld. Theoretical considerations on the motion of salt and fresh water. In Proc. of the Minn. Int. Hydraulics Conv., Joint meeting IAHR and Hyd. Div. ASCE., 321-333, 1953

[25] E.F. Toro, S.J. Billett. Centred TVD schemes for hyperbolic conservation laws. IMA J. Numer. Anal. 20(1): 47-79, 2000. MR1736950 (2001h:65097)

[26] E.F. Toro, A. Siviglia. PRICE: Primitive centred schemes for hyperbolic systems. Int. Jour. Num. Meth. Fluids, 42: 1263-1291, 2003. MR1994077

[27] E.F. Toro, V.A. Titarev. MUSTA fluxes for systems of conservation laws. J. Comput. Phys. 216(2): 403-429, 2006. MR2235378

[28] I. Toumi. A weak formulation of Roes approximate Riemann solver. J. Comput. Phys. 102(2): 360-373, 1992. MR1187694 (93h:65126)

Universidad de Málaga, Departamento Análisis Matemático, Campus de Teatinos s/n, 29071 Málaga, Spain

E-mail address: castro@anamat.cie.uma.es

Universidad de Málaga, Departamento Análisis Matemático, Campus de Teatinos s/n, 29071 Málaga, Spain

E-mail address: pardo@anamat.cie.uma.es

Universidad de Málaga, Departamento Análisis Matemático, Campus de Teatinos s/n, 29071 Málaga, Spain

E-mail address: pares@anamat.cie.uma.es

University of Trento. Laboratory of Applied Mathematics. Faculty of Engineering, 38050 Mesiano di Povo, Trento, Italy

E-mail address: toroe@ing.unitn.it 